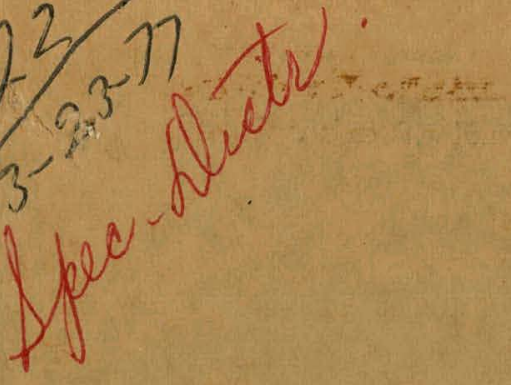

\author{
WAPD-TM-1288 \\ ERDA RESEARCH AND \\ DEVELOPMENT REPORT
}

\title{
MONTE CARLO SIMULATION USING THE METER SYSTEM WITH APPLICATIONS RELATED TO LWBR \\ (LWBR Development Program)
}

B. R. BEAUDOIN

FEBRUARY 1977

CONTRACT EY-76-C-11-0014

BETTIS ATOMIC POWER LABORATORY, WEST MIFFLIN, PA., OPERATED FOR THE U.S. ENERGY RESEARCH AND DEVELOPMENT ADMINISTRATION BY WESTINGHOUSE ELECTRIC CORPORATION 


\section{DISCLAIMER}

This report was prepared as an account of work sponsored by an agency of the United States Government. Neither the United States Government nor any agency Thereof, nor any of their employees, makes any warranty, express or implied, or assumes any legal liability or responsibility for the accuracy, completeness, or usefulness of any information, apparatus, product, or process disclosed, or represents that its use would not infringe privately owned rights. Reference herein to any specific commercial product, process, or service by trade name, trademark, manufacturer, or otherwise does not necessarily constitute or imply its endorsement, recommendation, or favoring by the United States Government or any agency thereof. The views and opinions of authors expressed herein do not necessarily state or reflect those of the United States Government or any agency thereof. 


\section{DISCLAIMER}

Portions of this document may be illegible in electronic image products. Images are produced from the best available original document. 
WAPD-TM- 1288

Special External Distribution

MONTE CARLO SIMULATION USING THE METER SYSTEM

WITH APPLICATIONS RELATED TO LWBR

(LWBR Development Program)

B. R. Beaudoin

February 1977

CONTRACT EY-76-C-11-0014

Printed in the United States of America

Available from the

National Technical Information Service

U. S. Department of Commerce

5285 . Port Royal Road

Springfield, Virginia 22151

\section{NOTE}

This document is an interim memorandum prepared primarily for internal reference and does not represent a final expression of the opinion of Westinghouse. When this memorandum is distributed externally, it is with the express understanding that Westinghouse makes no representation as to completeness, accuracy, or usability of information contained therein.

BETTIS ATOMIC POWER LABORATORY

WEST MIFFLIN, PENNSYLVANIA

Operated for the U. S. Energy Research and Development Administration by WESTINGHOUSE ELECTRIC CORPORATION

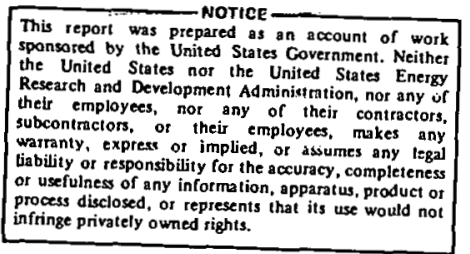


1.

This report was prepared as an account of work sponsored by the United States Government. Neither the United States, nor the United States Energy Research and Development Administration, nor any of their employees, nor any of their contractors, subcontractors, or their employees, makes any warranty, express or implied, or assumes any legal liability or responsibility for the accuracy, completeness or usefulness of any information, apparatus, productor process disclosed, or represents that its use would not infringe privately owned rights. 
Page

I. IIPIRODUCTION . . . . . . . . . . . . . . . . . I

II. DESCRIPTION OF METER SYSTEM ... . . . . . . . . . . . 3

A. General Purpose Routines in MEIER . . . . . . . . . 3

1. Input Routines............. 4

2. Random Number Generator Routines ........ 5

3. Output Routines .............. 8

B. Testing the Random Number Generator in NETER . . . 10

C. Use of METER to Program a Simulation . . . . . . . . 26

1. Operational Procedure . . . . . . . . 26

2. Sample Problem 1.............. . 34

3. Sample Problem 2............. 47

4. Sample Problem 3............. 53

D. Suggestions for Verifying Simulations.......... 61

III. SOME METER APPLICATIONS RELATED TO LWBR . . . . . . . . $6 I$

A. Fuel Rod Binary $\left(\mathrm{UO}_{2}-\mathrm{ThO}_{2}\right)$ Stack Lengths . . . . . 62

B. Fuel Rod Fissile Content .............. 62

C. Thoria Shim Pellets .............. 63

D. Flow Area Between Fuel Rods . ... . . . . . . 63

IV. SUMMARY ............................ 64

ACKNOWLEDGEMENTS ................. 65

REFERENCES ...................... 65

COMPUTER PROGRAM ABSTRACT ...................... 66 
METER is a Monte Carlo computer program which can be used to simulate the interaction between independent random variables and their effects on one or more dependentrandom variables. The program is easy to use for simple simulations but is capable of accommodating complex simulations. METER processes input, generates random numbers from several common frequency distributions under user control, performs the simulation which the user has coded in FORTRAN, and displays results.

\section{MONIE CARLO STMULATION USING THE METER SYSTEM}

WITH APPLICATIONS RELATED TO LWBR

(LWBR Development Program)

B. R. Beaudoin

\section{INIRODUCTION}

The design and manufacture of a nuclear plant and a reliable assessment of performance characteristics must take into account effects of design models, projected plant operating conditions, uncertainties in physical properties, and variations in as-manufactured components. Design models can be tried, tested, and perhaps compared to experiments. Operating conditions, physical properties, and component dimensions can be varied in the computer codes used to calculate plant performance, thereby producing sensitivities to important variables. This method of applying all uncertainties and tolerances multiplicatively will produce estimates of ranges of expected performance, for example, minimum and maximum expected core lifetime. In many instances such estimates will be overly pessimistic or optimistic, especially where many variables are involved, since they do not account for the random . 
behavior of variables. To make probablistic statements about performance-for example, the probability that core lifetime will be at least some given value-requires a statistical approach.

The statistical approach to assessing the effects on dependent variables of various independent random variables generally assumes that the random variables are normally distributed. For example, if $C=A+B$ where the two random variables $A$ and $B$ are normally distributed with means $A$ and $B$ and variances $v_{A}$ and $v_{B}$ (standard deviation $\sigma=\sqrt{\mathrm{v}}$ ), we would conclude that the dependent variable $C$ is nomally distributed with mean $C=A+B$ and variance $v_{C}=v_{A}+v_{B}$. Suppose, however, that only part of the A population went into producing $C$; for example, suppose that values less than $A_{1}$ and greater than $A_{2}$ were discarded as is frequently done by tolerancing. Further, suppose that the distribution of B was skewed. In this case, how would we characterize the distribution of $\mathrm{C}$ in simple analytical terms?

In order to evaluate the effects of nonnormal distributions and to evaluate complex interactions between and among many variables influencing the deslgil, manufacture, and operation of the Light Water Breeder Reactor (LWBR) the METER (Monte-Carlo Employed To Evaluate Responses) program was developed. METER began as a multi-option program to deal with specific problems related to LWBR. Each Monte Carlo simulation was executed by requesting a particular option in the program. As more uses were identified for the concept of Monte Carlo simulation, ME'ER became cumbersome to maintain. The multi-option system was dropped in favor of a general purpose system. This report describes a tool called MEIER which processes input, generates random numbers, and displays results. How the random numbers interact to form a Monte Carlo simulation is supplied by the user in the form of a FORIRAN subroutine. The FORTRAN subroutine can be as simple as: 


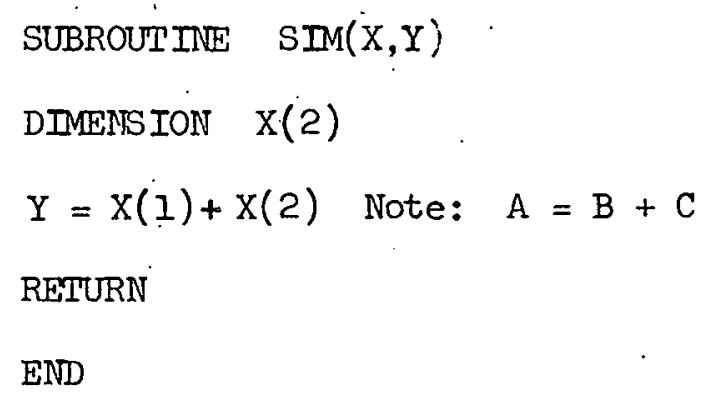
presented which explain examples of the use of METER and its application to the LWBR development effort and suggestions are given on methods for verifying METER Monte Carlo simulations.

II. DESCRIPTION OF METER SYSTEM

METER is a system of subroutines and functions which is almost a complete computer program. One subroutine, SIM, is referenced in MEIER by the FORTRAN statement

$$
\operatorname{CAIL} \operatorname{SIM}\left(\arg _{1}, \arg _{2}\right)
$$

and is supplied by the user. The user must write his. Own version of STM in such $a$ way that the subroutine accepts one or more independent variables referenced by $\arg _{1}$ and computes one or more dependent variables referenced by $\arg _{2}$. METER calls SIM repeatedly, supplying random values for the independent variables, and saves the resulting dependent variable values for later evaluation. Thus, by providing the SIM subroutine, the user completes the. METER program allowing it to perform a specific Monte Carlo simulation.

\section{A. General Purpose Routines in METER}

To perform a Monte Carlo simulation using METER one need only supply computer control cards for munning a METER job, a SIM subroutine (In FORTRAN), input cards describing the independent variable distributions and input cards 
labeling output. METER's general purpose routines will process the input, generate random independent and dependent values, and display output distributions.

II. A. 1. Input Routines

During the execution of METER, input is processed using the Bettis Input Package (reference 1) which assumes numbered cards containing data fields separated by commas; for exaraple,

$$
101,6, \mathrm{ABC}, 1.0
$$

Input cards describing the distributions needed by a MEIER similation are read by a subroutine in METER called DATAI. METER calls DATAI at the start of each simulation to read cards in the ISS series. The format of the ISS cards will be described in detail in section II.C.I. While typical uses of METER will not require calls on DATAl in the user's SIM subroutine, DATAI will be described here in the event it is required for particular applications.

Each call on DATAl results in the reading of a specified input card. The arguments of DATAI in their required order are C - on entry, the flaating point number of the card to be read. N3 - on return, the starting location in bjank common for the data on card $C$. MEIER uses blank common starting at the first location for storage of input card data; that is, the array Il needed by the subroutine INP (ref. I) is declared as blank. common.

N4 - on return, the mumber of entries found on card C. 
IIDI - on entry, the starting location in blank common for the distribution parameters required by METER's random number generator (described in more detail in section II.A.2).

XNOM - on return, nominal value for the random variable whose distribution is described on card $\mathrm{C}$.

NER - on return, unchanged if card $C$ was correct; set to 1 if card $C$ was in error.

As stated previously, the typical user will not need to program direct calls on DATAI in his STM subroutine provided input card series ISS is used for describing input distributions.

II. A. 2. Random Number Generator Routines

The random number generator in MEIER consists of five FORTRAN functions and subroutines and permits random numbers to be selected from six types of distributions. These are:

1. Flat or uniform - all random values of $x_{\text {between }} x_{1}$ and $x_{2}$ are equally probable.

2. Line - frequency distribution for the random values is a straight line (note: the flat distribution is equivalent to a line distribution with zero slope).

3. Normal - frequency distribution for the random values is the Gaussian or bell shaped curve with specified mean and standard deviation and possibly blased and/or truncated.

4. Point - all random values are set to a specified constant (useful when setting random variables to constants without re-programming SIM or for verifying a simulation). 
5. Chi-square - frequency distribution for the random values is skewed either toward low values or toward high values.

6. Histogram - frequency distribution for the random values is a histogram or step function with any specified shape.

Exactly how these distributions are specified on input cards in a METER simulation is described in section II.C.I.

The five functions and subroutines which comprise the random number generators are named INIIRV, RV, RANVAR; FUNCTN, and NEWBEQ. Ty pleal METER Mumle Carlu slmulations will not require the user to program direct calls on the random number routines. METER performs that function automatically during each repetition. The five routines will be described here in case the user finds a need to call them directly.

\section{SUBROUTINE IVIIRV(TA, RA)}

INIIRV initializes the parameters for a distribution; that is, INIIRV uses the input which specified the particular distribution to compute constants needed when RANVAR is requested to supply the next random number from that distribution. IA and RA are equivalenced arrays containing the input and the constants for the distribution. When reading the ISS cards METER makes sequential calls on DATAI (section II.A.I) and INIIRV. For example the statements

$$
\begin{aligned}
& C=101 \\
& \text { CALI DATAI(C, N3, N4,I, XNOM,XTOL, NER) } \\
& \text { CALL INIIRV(IA (I), RA(I)) }
\end{aligned}
$$


would result in initialization of the distribution for the first independent random variable in a METER mun.

\section{FUNCTION RV(I)}

RV returns the next random value from the $I^{\text {th }}$ distribution provided DATAI and INIIRV have been called for the $I^{\text {th }}$ distribution. For example the statement

$$
\mathrm{X}=\mathrm{RV}(1)
$$

would place the next random value for the first distribution (described on input card 10I) into the FORTRAN variable $X$.

FUNCTION RANVAR(IA, RA)

RANVAR computes the next random number for the distribution whose parameters are stored in the equivalenced arrays IA and RA. The function RV uses RANVAR.

FUNCTION FUNCTN( IA, RA, NOP, $X$ )

Given the distribution parameters stored in equivalenced arrays IA and RA, NOP - the type of distribution ( 1 through 6 as defined earjier) and $X$-an independent variable value-FUNCTN computes the value of the dependent variable $-y=f(x)$. FUNCTN is used by INIIRV and RANVAR. SUBROUTINE NEWSER

RANVAR uses the function RANDOM in the FORTRAN library when generating its random variables. RANDOM generates values between 0 and $I$ with equal probability (flat distribution between $U$ and 1 ). Given a 
starting number, RANDOM will always generate the same set of random numbers between $O$ and 1 . Each call on METER's subroutine NEWSEQ initiates a new start in RANDOM; that is, a new set of random numbers. This capability is important for correlated sampling when testing the effects of distributions on results.

The routines discussed above constitute the random mumber generator in MNTFR whirh provides random numbers from several types of distributions. The typical user should have no need to make direct calls on these routines; however, the above descriptions should suffice for more sophisticated simulations where direct calls might be required.

\section{A. 3. Output Routine}

output from a METER Monte Carlo simulation is displayed in tabular form and in graphical form by a subroutine called DISPLA. As is the case with the input and random number routines, DISPLA need not be called in the typical user's 3 MM subroutine. METER automatically calls DISPLA to compute the mean, variance, and skewness', to tabulate a histogram and to plot the histogram for each output or dependent variable distribution or array of results. The arguments of DISPIA are: NOP - on entry, must be set to $O$ to indicate that DISFLA is being called Irom MEI'tK.

NDATA - on entry, the size of the population being displayed. DATA - on entry, the array containing the data being displayed. NCLASS - on entry, the mumber of classes or steps in the output histogram. 
STEP - on entry, step size for histogram if NCLASS $=0$.

A - working array of size NCLASS + 1 .

$B$ - working array of size NCLASS.

C -' working array of size NCLASS.

OTILF - on entry, 6-word array for display title (taken from ass

cards in METER).

UNIIS - on entry, 1-word containing the units for the output distribution (taken from $35 S$ cards in METER).

PIIILE - on entry, 3-word array for the plot title (taken from 4SS cards when DISPLA is called from METER).

COMMNT - on entry, 1-word plot comment (not used when DISPLA is called from METER).

$X I A B E L$ - on entry, 3-word X-axis plot label (set to the character string in UNIIS (above) when DISPLA is called from METER.

YIABEL - on entry, 3 word Y-axis plot label (set to the character string RELATIVE FREQUENCY in METER.

VAINOM - on entry, nominal value of the dependent variable whose distribution is being displayed. Computed in METER using the nominal values for the independent variables and the user's SIM routine which defines how to compute the variable.

SIGMA - on entry, number of standard deviations at which to truncate the output plot (read from input card 0 or set to 4.0 if card 0 is not input). 
IO - on entry, unit number for WRITE statements (METER executes CAJI OÜTIAP(IO) to obtain IO).

XMIN - on return, smallest value in DATA.

XMAX - on return, largest value in DATA.

XMEAN - on return, mean value of the NDATA values in DATA.

VAR - nn return, variance of tho population.

STD - on return, standara deviation of lie purulation (STI $=\sqrt{\mathrm{VAR}}$ ).

SKEW - on return, coefficient of skewness of the population.

Use of input card 0 and of input card series 2SS, 3SS, and 4SS is described in detail in section II.C.I. Each plotted histogram will include a superimposed normal distribution based on the mean and variance of the data processed by DISPLA.

II. B. Testing the Random Number Generator in METER

As discussed in section II.A.2, the METER program contains a random number generator capable of producing random numbers from flve types of frequency distributions. The distributions available in METER are flat or uniform, line, normal, chisquare, and histogram. If a random variable is to be treated as a constant in certain cases, a point or constant option is available. The random number generator in METER permits great flexibility for defining random variables in Monte Carlo simulations. The capability to truncate or to bias normal distributions is especially useful in sensitivity studies. To demonstrate that METER's random number generator produces the requested distributions, $a$ testing routine was programmed and executed. The test results are displayed in the tables and figures on pages 12 through 25 . 
In each of the seven examples METER generated 100,000 random numbers and displayed the results in a histogram. The first example requested a flat distrioution of random numbers between 0.95 and $1.05(1.0 \pm .05)$. The input cards for defining the distributions using the Bettis Input Package are discussed in detail in section II.C. Table II.B.I contains the resulting histogram (2l classes) for the first example. The columns labeled "MINIMUM" and "MAXIMUM" define boundaries of each class. The column labeled "COUNTS" identifies the number of random values which fell into each class. For example, of the 100,000 random values generated, $4739 \mathrm{fell}$ into class 12 (1.00238 to 1.00714$)$. Note that a true flat or uniform frequency distribution should have $4762(100,000 / 21)$ values in each of the 21 classes. Class 6 has the fewest counts, (4682) and is low by $1.68 \%$. Class 14 with the most counts (4898) is high by $2.86 \%$. The column labeled "FRACTION" Iists. the fraction of total counts in each class. The columns labeled " $X$ ", "P(X)", and " $F(X)$ " relate to the values plotted in Figure II.B.1. " $X$ " is the midpoint of the class. " $P(X)$ ' is the plotted relative number of counts or relative frequency for the class normalized to unit area under the histogram. "F(X)" is the trie value of the requested frequency distribution function at " $\mathrm{X}$ " also normalized to unit area. Thus, Figure II.B.I graphically displays METER's frequency distribution for the 100,000 random numbers along with the requested frequency distribution.

Tables II.B.2 through II.B.7 and figures. II.B.2 through II.B.7 display results for examples 2 through 7. The tables and figures for these examples are similar to those for the first example. Each example produces random 
RAKDOM NUMBER GEIERATOR EXAIPLE 1

TEST OF RAFDOY IUIBER GENERATOR IN WETER

EXAMPLE 1: 101, FLAT, 1,0,0.05

\begin{tabular}{|c|c|}
\hline $\begin{array}{r}\text { CLASS } \\
1 \\
2 \\
3 \\
4 \\
5 \\
6 \\
7 \\
8 \\
9 \\
10 \\
11 \\
12 \\
13 \\
14 \\
15 \\
16 \\
17 \\
18 \\
19 \\
20 \\
21 \\
\text { AL }\end{array}$ & 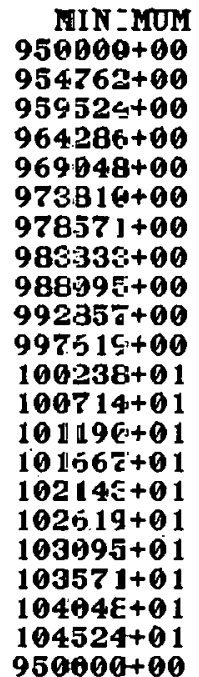 \\
\hline
\end{tabular}

\begin{tabular}{|c|}
\hline $\begin{array}{r}\text { MAXIMUM } \\
954762+00 \\
959524+00 \\
964286+00 \\
969048+00 \\
973810+00 \\
978571+00 \\
983333+00 \\
988095+00 \\
992857+00 \\
997619+00 \\
100238+01 \\
100714+01 \\
101190+01 \\
101667+01 \\
102143+01 \\
102619+01 \\
103095+01 \\
103571+01 \\
10404 B+01 \\
104524+01 \\
105000+01 \\
10500 D+01\end{array}$ \\
\hline
\end{tabular}
$471200-01$ $472100-0$ $4 B 1500-01$ $472400-01$ $477100-0$ 458200-0 $459700-01$ 472200-01 478200-01 $436600-01$ $459600-0$ $473900-01$ 476800-01 $489800-0$ $474700-6$ $4 ? 1200-0$ $4 ? 1400-0$ $486600-0$ $4 ? 8200-0$ $485800-01$ $4.2800-01$
$100000+01$
HETE

$X$
$952381+00$
$957143+00$
$961965+00$
$966667+00$
$971429+00$
$976190+00$
$980952+00$
$985714+00$
$990476+00$
$995238+00$
$100000+01$
$100476+01$
$100952+01$
$101429+\theta 1$
$101905+\theta 1$
$102381+01$
$102857+01$
$103333+01$
$103810+01$
$104286+01$
$104762+01$

\begin{tabular}{|c|c|}
\hline 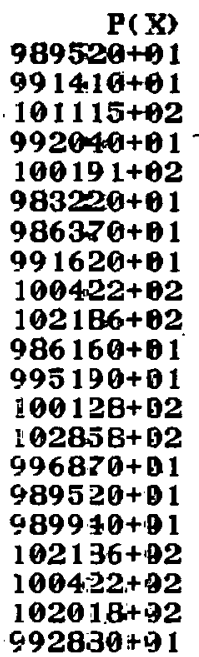 & $\begin{array}{r}F(X: \\
100000+02 \\
100000+02 \\
100000+02 \\
100000+02 \\
100000+02 \\
100000+02 \\
100000+02 \\
100000+02 \\
100000+02 \\
100000+02 \\
100000+02 \\
100000+02 \\
100000+02 \\
100000+02 \\
100000+02 \\
100000+02 \\
100000+02 \\
100000+02 \\
100000+02 \\
100000+02 \\
100000+02\end{array}$ \\
\hline
\end{tabular}

INRAN $=$ B8290595182968 


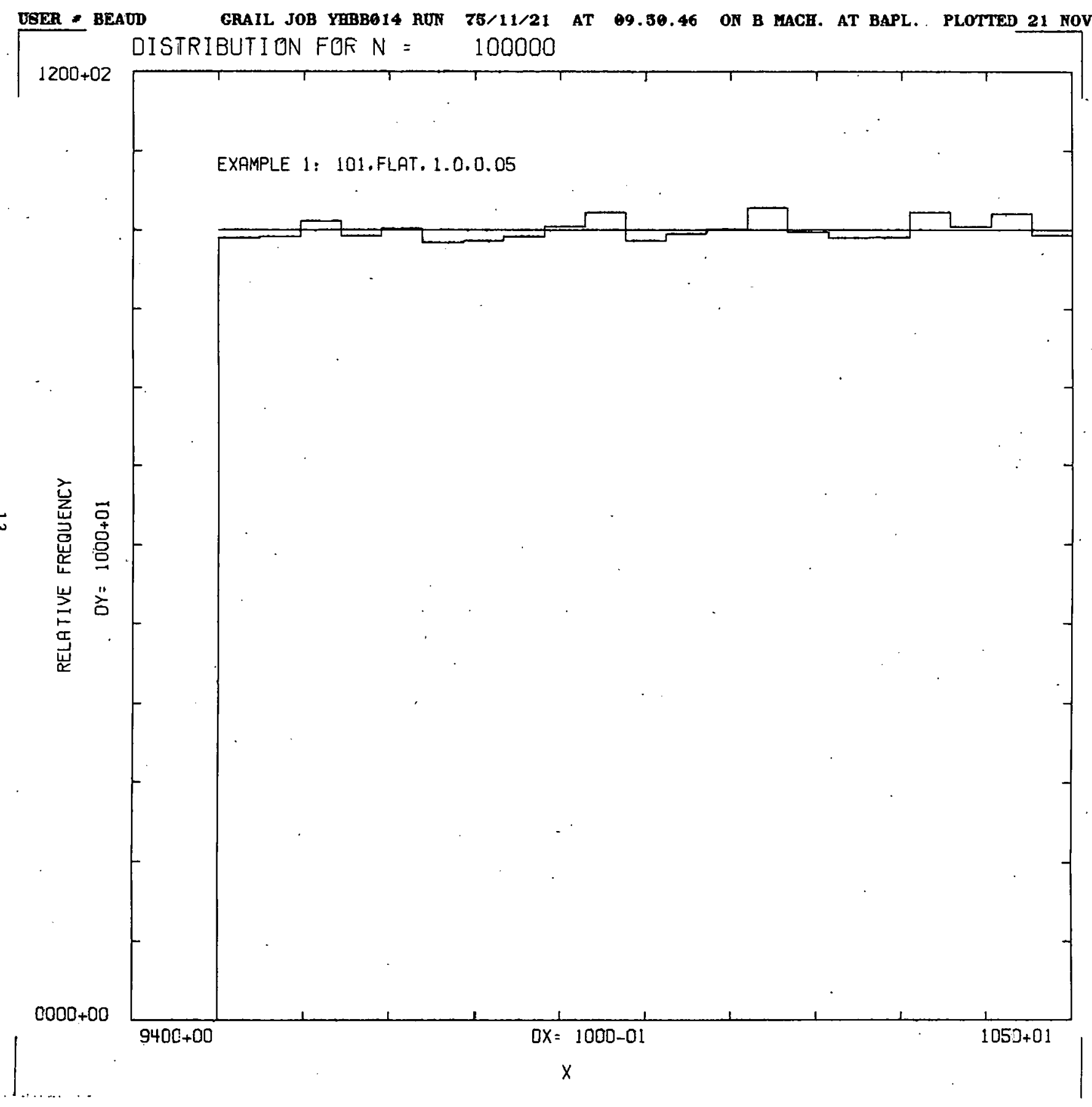


TEST OF RANDOM NUMBER GENERATOR II METER

EXAMPLE 2: 101, LINE, 200.0,-189,9,1.0,0.05

$\begin{array}{rr}\text { CLASS } & \text { MIIIMUM } \\ 1 & 950000+00 \\ 2 & 954762+00 \\ 3 & 957524+00 \\ 4 & 964286+00 \\ 5 & 969048+00 \\ 6 & 973820+00 \\ 7 & 978571+00 \\ 8 & 983333+00 \\ 9 & 988095+00 \\ 10 & 992857+00 \\ 11 & 9976 \leq 9+00 \\ 12 & 16 B 238+01 \\ 13 & 16 B 754+01 \\ 14 & 161190+01 \\ 15 & 161667+01 \\ 16 & 162143+01 \\ 17 & 162619+01 \\ 18 & 163095+01 \\ 19 & 163571+01 \\ 20 & 164048+01 \\ 21 & 164524+01 \\ \text { ALL } & 950000+00\end{array}$

HAXIMUM
$954762+00$
$959524+00$
$964286+00$
$969048+00$
$973810+00$
$978571+00$
$983933+00$
$988095+00$
$992857+00$
$997619+00$
$100238+01$
$100714+01$
$101190+01$
$101667+01$
$102143+01$
$102619+01$
$103095+01$
$103571+01$
$104048+01$
$104524+01$
$105000+01$
$105000+01$

COUNTS
274
779
1125
1602
2031
2463
3021
3389
3835
4330
4719
5111
5617
6186
6572
6961
7575
7825
8360
8919
9306
100006

FRACTION
$274000-02$
$779000-02$
$112500-01$
$160200-01$
$203100-01$
$246300-01$
$302100-01$
$338900-01$
$383500-01$
$433000-01$
$471900-01$
$511100-01$
$561700-01$
$618600-01$
$657200-01$
$696100-01$
$757500-01$
$782500-01$
$836000-01$
$891900-01$
$930600-01$
$100000+01$

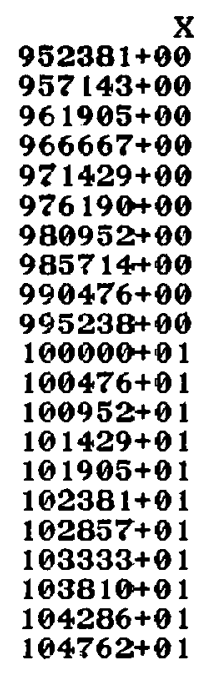

\begin{tabular}{|c|c|}
\hline $\begin{array}{r}P(X) \\
575460+0 Q \\
163590+01 \\
236250+01 \\
336420+01 \\
426510+01 \\
517230+01 \\
634410+01 \\
711690+01 \\
805350+01 \\
909390+01 \\
990990+01 \\
107331+02 \\
117957+02 \\
129966+02 \\
138012+02 \\
146181+02 \\
159075+02 \\
164325+02 \\
175560+02 \\
187299+02 \\
195426+02\end{array}$ & 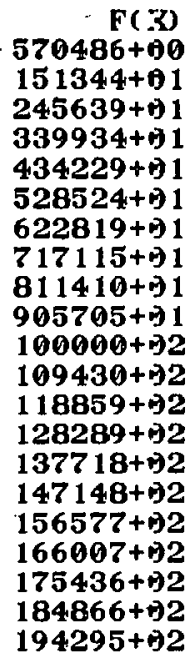 \\
\hline
\end{tabular}


USER - BEAUD GRAIL JOB YHBBO 14 RUI 75/11/21 AT 09.50.46 ON B MACH. AT BAPL. PLOTTED 21 NOV 25 AT 10:43:3 FRAME O02

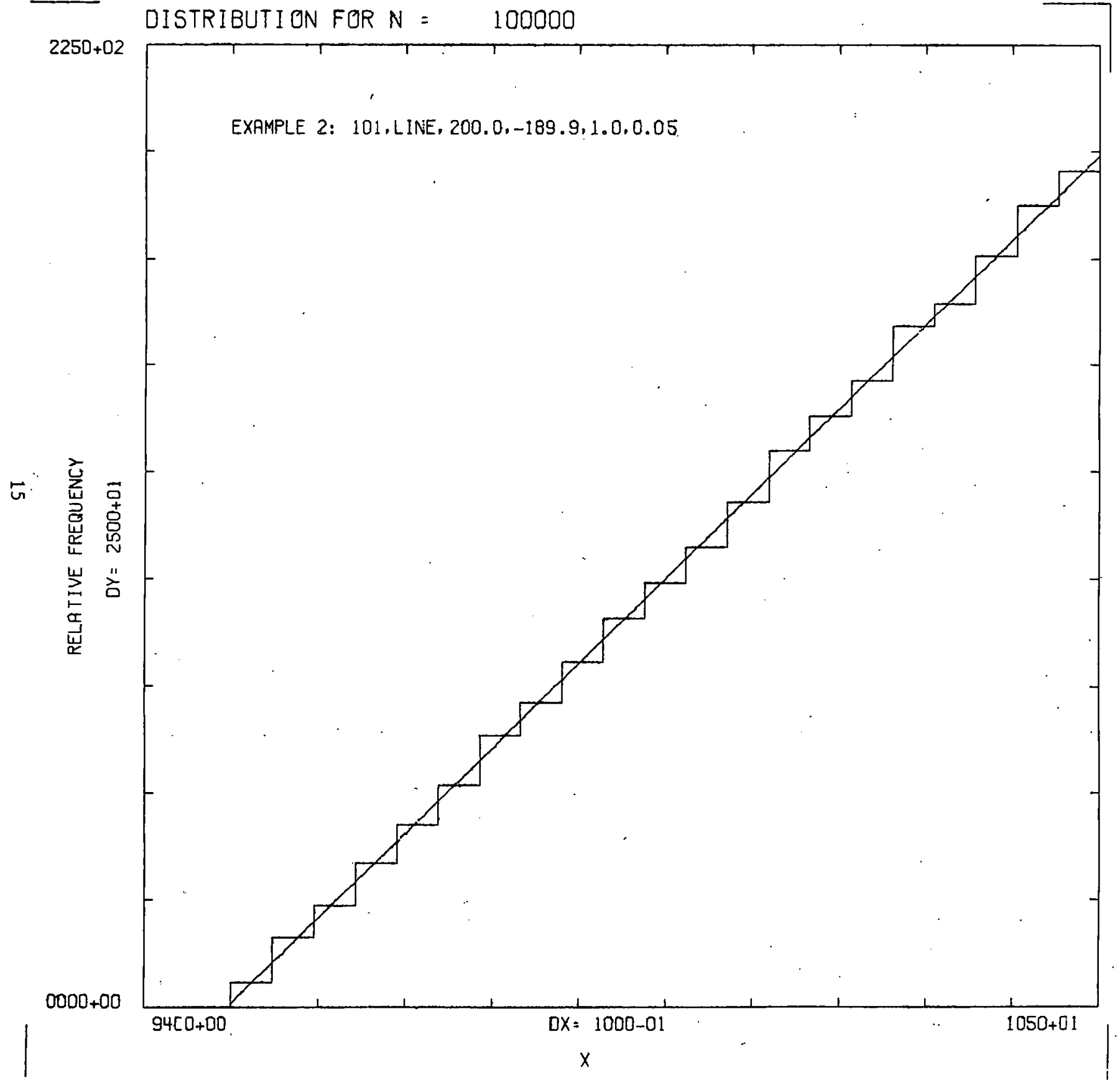


RANDOM NUMBER GEMERATOR EXAMPLE 3

TEST OF RANDOM IUMBER GENERATOR IN METER

EXAMPLE 3: 191, NORMAL, $1.0,0.0125,1.0,0.05$

$\begin{array}{rr}\text { CLASS } & \text { M1SIMUM } \\ 1 & 950060+00 \\ 2 & 954762+00 \\ 3 & 959524+00 \\ 4 & 964266+00 \\ 5 & 969048+00 \\ 6 & 973810+00 \\ 7 & 978571+00 \\ 8 & 983393+00 \\ 9 & 988095+00 \\ 10 & 992857+00 \\ 11 & 997619+00 \\ 12 & 100238+01 \\ 13 & 100714+01 \\ 14 & 101190+01 \\ 15 & 101667+01 \\ 16 & 102143+01 \\ 17 & 102619+01 \\ 18 & 103095+01 \\ 19 & 103571+01 \\ 20 & 10 \leq 048+01 \\ 21 & 104524+01 \\ \text { ALL } & 956000+00\end{array}$

\begin{tabular}{|c|c|c|c|}
\hline $\begin{array}{r}\text { KAXIMUM } \\
954762+00 \\
959524+00 \\
964286+00 \\
969048+00 \\
973810+00 \\
978571+00 \\
9 B 3333+00 \\
988095+00 \\
972857+00 \\
997619+00 \\
190238+01 \\
190714+01 \\
191190+01 \\
191667+01 \\
192143+01 \\
192619+01 \\
193095+01 \\
193571+01 \\
104048+01 \\
104524+01 \\
105000+01 \\
105000+01\end{array}$ & $\begin{array}{r}\text { COLINTS } \\
8 \\
43 \\
169 \\
435 \\
1252 \\
2443 \\
4740 \\
7965 \\
11268 \\
14160 \\
15033 \\
13981 \\
11378 \\
8061 \\
4799 \\
2568 \\
1131 \\
427 \\
139 \\
48 \\
12 \\
100000\end{array}$ & $\begin{array}{r}\text { FRACTION } \\
800000-04 \\
430000-03 \\
169000-02 \\
435000-02 \\
125200-01 \\
244300-01 \\
474000-01 \\
796500-01 \\
=12680+00 \\
141600+00 \\
150330+00 \\
139810+00 \\
113780+00 \\
800100-01 \\
479900-01 \\
256800-01 \\
113100-01 \\
427000-02 \\
139000-02 \\
480000-03 \\
120000-03 \\
100000+01\end{array}$ & $\begin{array}{l}952381+00 \\
957143+00 \\
961905+00 \\
966667+00 \\
971429+00 \\
976190+00 \\
980952+00 \\
985714+00 \\
990476+00 \\
995238+00 \\
100000+01 \\
109476+01 \\
100952+01 \\
101429+01 \\
101905+01 \\
102381+01 \\
102857+01 \\
103333+01 \\
103810+01 \\
104286+01 \\
104762+01\end{array}$ \\
\hline
\end{tabular}

$P(X)$
$168000-01$
$903900-01$
$354900+00$
$913500+00$
$262920+01$
$513030-01$
$995400-01$
$167265+02$
$23662 B+02$
$297369+02$
$315693+02$
$293601+02$
$238933+02$
$168921+02$
$100779+02$
$53939+01$
$237519+01$
$896093+00$
$291509+00$
$100809+00$
$25260 \theta-01$

$225260-0$

894210-01

$307021+00$

$911737+00$

$234177+01$

$520226+0$

$520226+0$

$166114+02$

$166114+02$

$09634+0$

$296834+02$

$319174+02$

$296834+02$

$238767+02$

$166114+02$

$999569+01$

$520226+0$

$234177+01$

$911737+03$

$307021+03$

894210-0

225260-01 
DGER - BEAUD GRAIL JOB YHBBO 14 RUT 75/11/21 AT 69.50.46 ON B MACH. AT BAPL. PLOTTED 21 NOV 73 AT 10:44: 19 FRAME 003

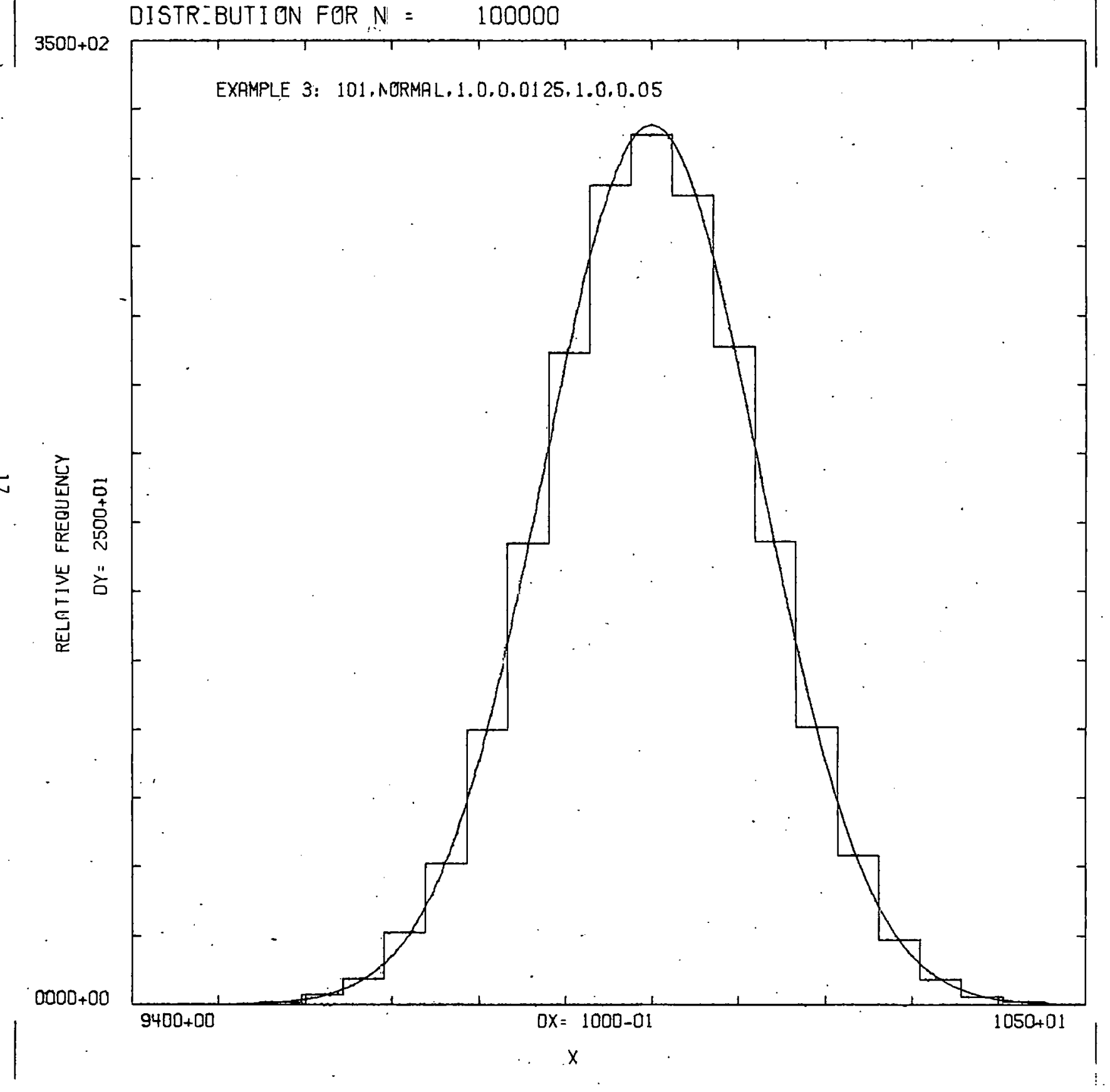


TEST OF RANDOM NUMBER GENERATOR IN METER

- EXAMPLE 4: 101, NORMAL, 1.025,0.025,1.0,9.05

\begin{tabular}{|c|c|c|c|c|}
\hline $\begin{array}{r}\text { CLASS } \\
1 \\
2 \\
3 \\
4 \\
5 \\
5 \\
6 \\
7 \\
8 \\
9 \\
10 \\
11 \\
12 \\
13 \\
14 \\
15 \\
16 \\
17 \\
18 \\
19 \\
20 \\
21 \\
\text { ALL }\end{array}$ & $\begin{array}{r}\text { MN I FUM } \\
950000+00 \\
954762+00 \\
959524+00 \\
964286+00 \\
969648+00 \\
973810+00 \\
978571+00 \\
983633+00 \\
988095+00 \\
992857+00 \\
997619+00 \\
100238+01 \\
100714+01 \\
101190+01 \\
101667+01 \\
102143+01 \\
102619+01 \\
103095+01 \\
103571+01 \\
104048+01 \\
104524+01 \\
950060+00\end{array}$ & $\begin{array}{r}\text { GAXIMUM } \\
954762+00 \\
959524+00 \\
964286+00 \\
969048+00 \\
975810+00 \\
978571+00 \\
986333+00 \\
986095+00 \\
992857+00 \\
996619+00 \\
109238+01 \\
100714+01 \\
101190+01 \\
101667+01 \\
102143+01 \\
102619+01 \\
103095+01 \\
103571+01 \\
164948+01 \\
104524+01 \\
105060+01 \\
105060+01\end{array}$ & $\begin{array}{r}\text { COUNTS } \\
119 \\
251 \\
333 \\
615 \\
9.57 \\
1312 \\
1843 \\
2693 \\
3598 \\
4519 \\
5317 \\
6591 \\
7438 \\
8373 \\
8878 \\
8968 \\
8714 \\
8697 \\
8099 \\
7156 \\
5947 \\
100064\end{array}$ & $\begin{array}{r}\text { FFACTION } \\
119000-02 \\
251000-02 \\
383000-02 \\
615000-02 \\
957000-02 \\
131200-01 \\
184300-01 \\
260800-01 \\
350800-01 \\
451900-01 \\
531700-01 \\
650100-01 \\
743900-01 \\
837300-01 \\
887800-01 \\
896300-01 \\
871400-01 \\
860700-01 \\
800900-01 \\
714700-01 \\
594700-01 \\
100000+01\end{array}$ \\
\hline
\end{tabular}

$X$
$952381+00$
$957143+00$
$961965+00$
$966667+00$
$971429+00$
$976190+00$
$980952+00$
$985714+00$
$990476+60$
$995238+00$
$100000+\theta 1$
$100476+01$
$109952+01$
$101429+01$
$101905+01$
$102381+01$
$102857+01$
$103333+01$
$103810+01$
$104286+01$
$104762+01$

\section{$\mathbf{X}$} 249900 $(X)$ $249900+00$ $527100+00$ $804300+00$ $129150+01$ $200970+01$ $275520+01$ $387030+01$ $547680 \div 1$ $736680-1$ 948990-01 111657-02 136521-0D 156093-02 175833-02 186438-02 $188328 \div 02$ $182994+\theta .2$ $180747+02$ $168000+02$ $150087+02$ $124.887+6.2$ 
OSER - BEAUD 、 GRAIL JOB YHBBO14 RUN 75/11/21 AT 09.50.46 ON B MACH. AT BAPL. PLOTTED 21 NOV 75 AT 10:45:22 FRAME 004

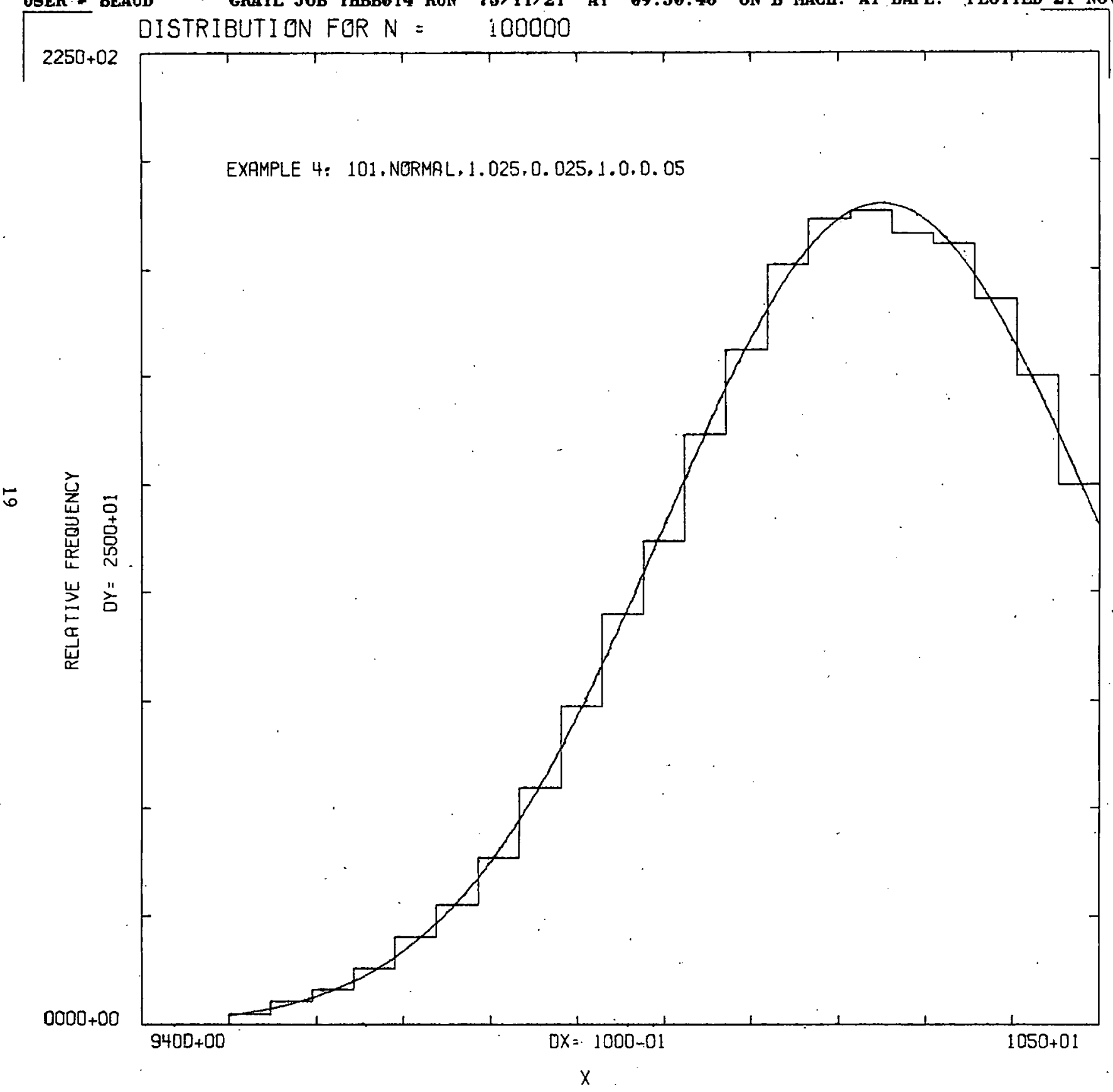


TEST OF HANLOM NMBER GENERATOR IN METER

EXAMPLE 5: 101, CHISQUARE, 0.95,0.96,3,1.0.0.05

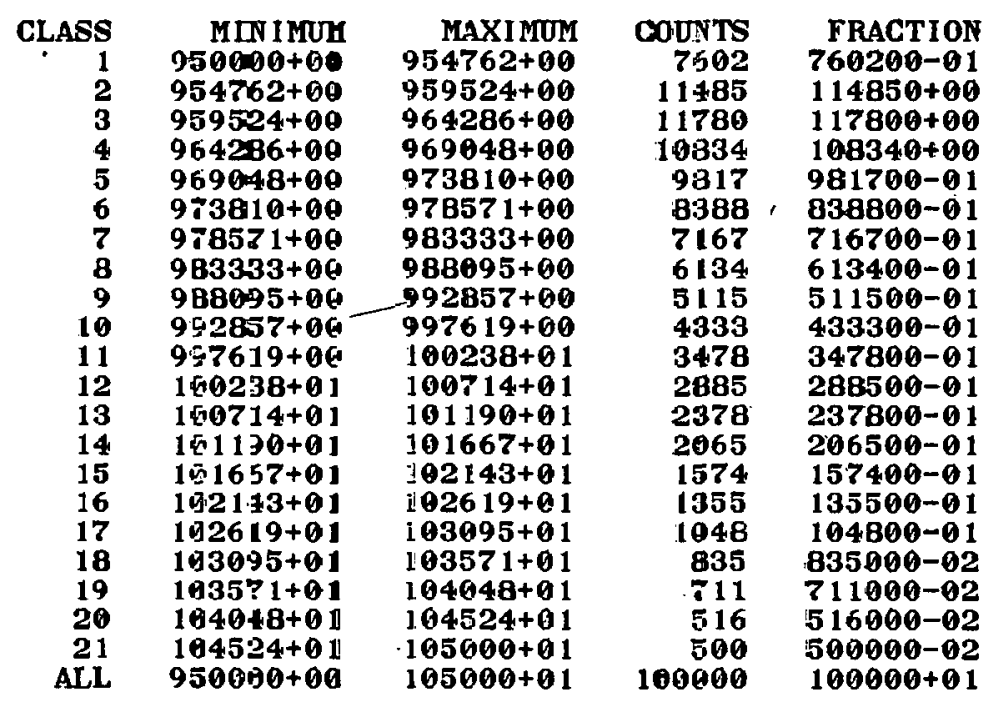

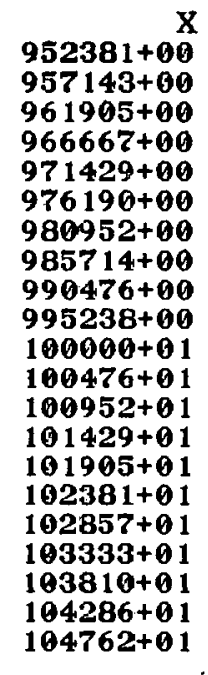

\begin{tabular}{|c|}
\hline 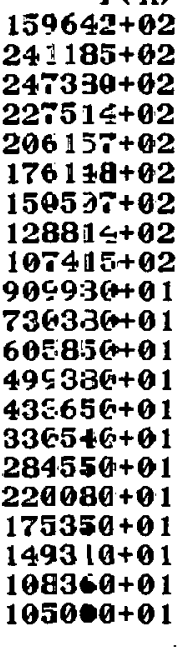 \\
\hline
\end{tabular}




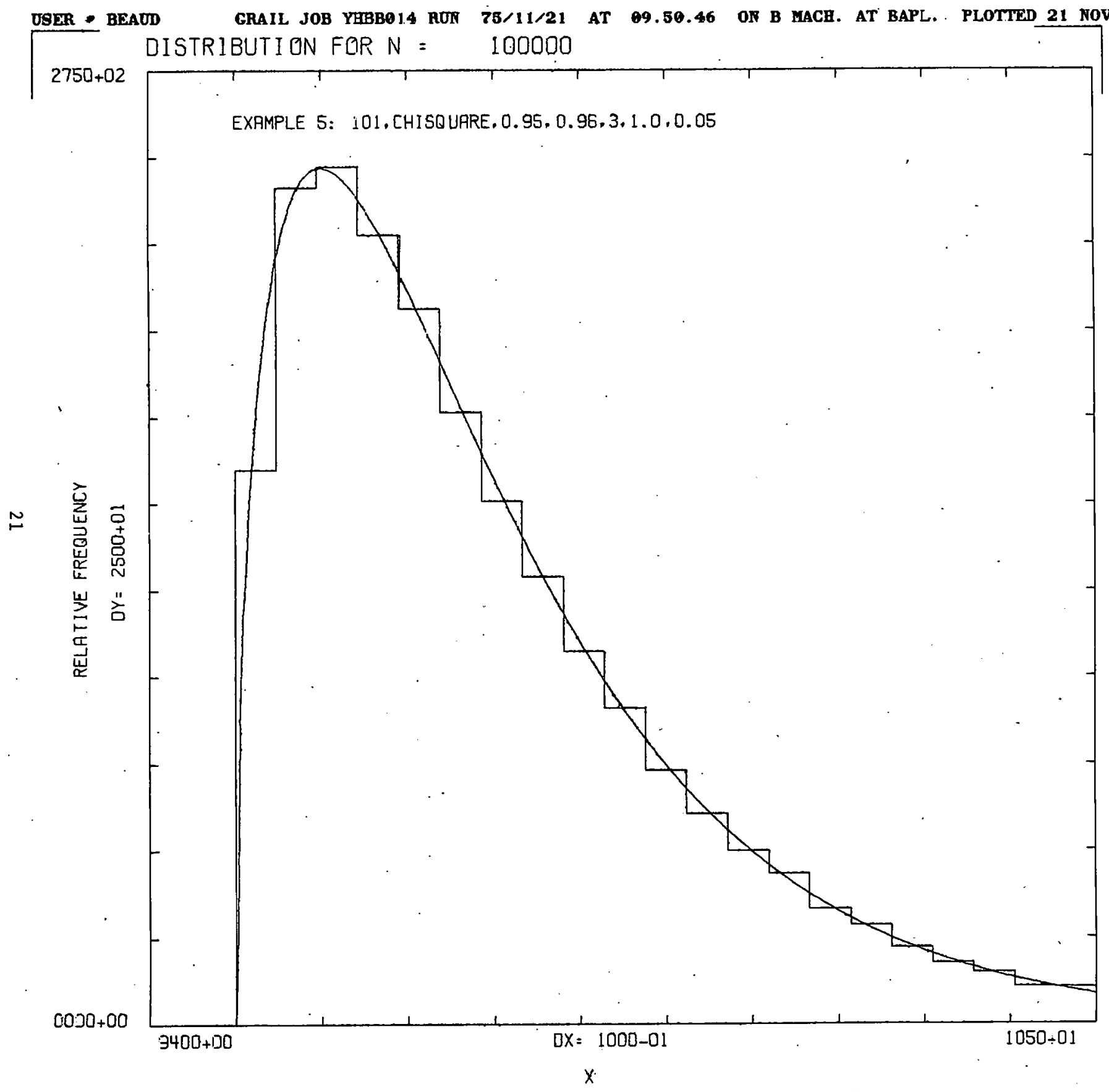


TEST OF RANDOM NUMBER GENERATOR IN METER

EXAMPLE 6: 1B1:HISTOGRMM, 3,0.95,0.03333,10.0,30.0,60.0,1.0,0.05

$\begin{array}{rr}\text { CLASS } & \text { MININUM } \\ 1 & 9506 D 0+00 \\ 2 & 954662+00 \\ 3 & 959524+00 \\ 4 & 964286+00 \\ 5 & 969648+00 \\ 6 & 973810+00 \\ 7 & 978571+00 \\ 8 & 983633400 \\ 9 & 988695+00 \\ 10 & 992657+00 \\ 11 & 997619+00 \\ 12 & 100238+01 \\ 13 & 100714+01 \\ 14 & 101190+01 \\ 15 & 101657+01 \\ 16 & 102143+01 \\ 17 & 102619+01 \\ 18 & 103095+01 \\ 19 & 103571+01 \\ 20 & 104048+01 \\ 21 & 104524+01 \\ \text { ALL } & 950090+00\end{array}$

$\begin{array}{rr}\text { COUNTS } & \text { FRACTION } \\ 1415 & 141500-01 \\ 1424 & 142400-01 \\ 1493 & 149300-01 \\ 1452 & 145200-01 \\ 1464 & 147400-01 \\ 1543 & 134300-01 \\ 1403 & 140300-01 \\ 4248 & 424809-01 \\ 4262 & 426200-01 \\ 4312 & 431200-01 \\ 4136 & 413600-01 \\ 4299 & 429900-01 \\ 4312 & 431200-01 \\ 4372 & 437200-01 \\ 8668 & 860800-01 \\ 8668 & 860800-01 \\ 8465 & 840500-01 \\ 8597 & 859700-01 \\ 8613 & 861300-01 \\ 8660 & 866060-01 \\ 8564 & 856400-01 \\ 100060 & 100006+01\end{array}$

$\mathrm{x}$
$952381+00$
$957143+00$
$961905+00$
$966667+00$
$971429+00$
$976190+00$
$980952+00$
$985714+00$
$990476+00$
$995238+00$
$100000+01$
$100476+01$
$100952+01$
$101429+01$
$101905+01$
$102381+01$
$102857+01$
$103333+01$
$103810+01$
$104286+01$
$104762+01$

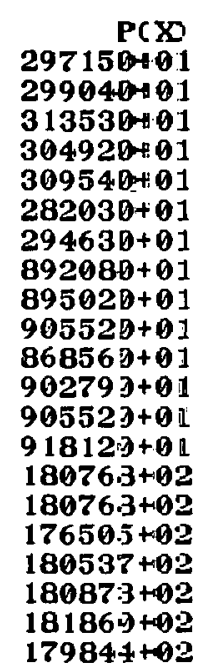

$F(X)$

$300030+01$

$300030+01$

$300030+01$

$300030+01$

$300030+01$

(1)

$300030+01$

$900090+61$

$90000+01$

$900090+01$

$900090+6$

$900090+01$

$900090+01$

$900090+01$

$180018+02$

$180018+02$

$180018+02$

$180018+02$

$180018+02$

$180018+02$

$180018+02$ 


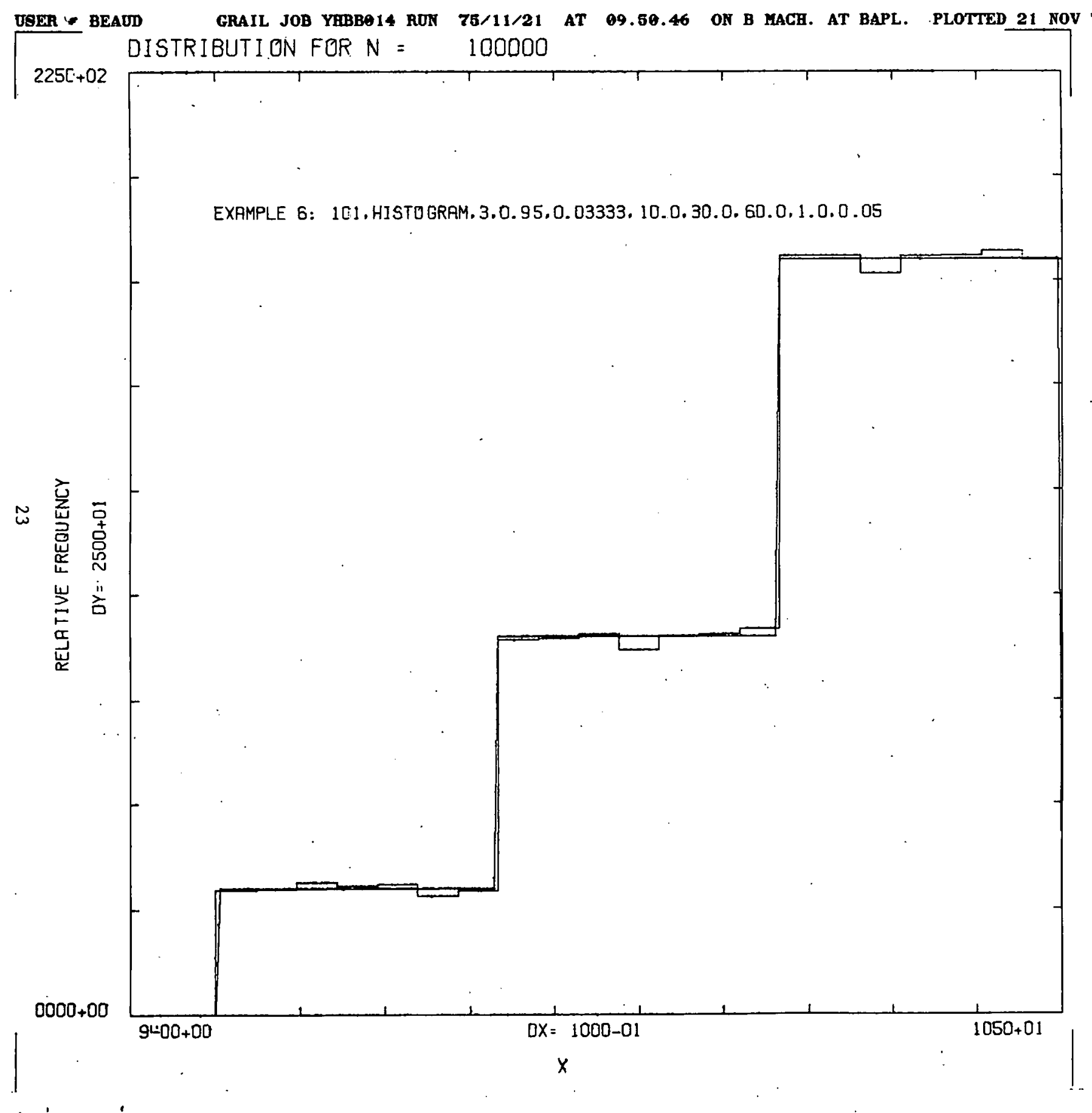


TEST OF RAYDOM NUYBFA GENERATOR IF NETER

EXAMPLE $7: \quad 161$, POINT $, 1.0,1.0,0.05$

$\begin{array}{rr}\text { CLASS } & \text { YINIMUM } \\ 1 & 9590 E 0+00 \\ 2 & 9547 E 2+00 \\ 3 & 959544+00 \\ 4 & 9642 E 6+00 \\ 5 & 969048+00 \\ 6 & 973810+00 \\ 7 & 978571+00 \\ 8 & 983333+00 \\ 9 & 988095+00 \\ 10 & 992857+00 \\ 11 & 997619+00 \\ 12 & 109238+01 \\ 13 & 109714+01 \\ 14 & 101190+01 \\ 15 & 101667+01 \\ 16 & 102143+01 \\ 17 & 102619+01 \\ 18 & 103095+01 \\ 19 & 163571+01 \\ 20 & 104048+01 \\ 21 & 104524+01 \\ \text { ALL } & 950000+00\end{array}$

$\begin{array}{rr}\text { MAXIMUM } & \text { COINTS } \\ 954762+00 & 0 \\ 959524+00 & 0 \\ 964286+00 & 0 \\ 969048+00 & 0 \\ 973810+00 & 0 \\ 978571+00 & 0 \\ 983333+00 & 0 \\ 988095+00 & 0 \\ 992857+00 & 0 \\ 997619+00 & 0 \\ 100238+01 & 100000 \\ 100714+01 & 0 \\ 101190+01 & 0 \\ 101667+01 & 0 \\ 102143+01 & 0 \\ 102619+01 & 0 \\ 103095+01 & 0 \\ 103571+01 & 0 \\ 104048+01 & 0 \\ 104524+01 & 0 \\ 105000+01 & 0 \\ 105000+01 & 100000\end{array}$

FRACTION $\rightarrow 00000+00$ $000000+00$ $000000+00$ $000000+00$ $000000+00$ $000000+00$ $000000+00$ $000000+00$ $000000+00$ $000000+00$ $100000+01$ $000000+00$ $000000+00$ $000000+00$ $000000+00$ $000000+00$ $00000+00$ $00000+0$ $000000+00$ $0000+0$ $000000+00$ $100000+01$

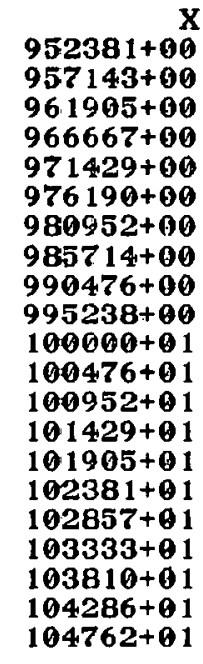

1.

$\begin{array}{rr}P(X) & F(X) \\ 000000+00 & 000000+(40\end{array}$ $000300+00 \quad 000000+60$ $000300+00 \quad 000000+60$ $000300+00000000+60$ $000300+00 \quad 000000+60$ $000300+00 \quad 000000+60$ $000300+00 \quad 000000+60$ $000500+00 \quad 000000+\mathrm{C} 0$ $000400+0 \theta \quad 000000+C 0$ $000000+00 \quad 000000+C 0$ $210 y 0 b+03 \quad 950000+C 2$ $000300+00 \quad 000000+C 0$ $000000+00 \quad 000000+00$ $000300+00 \quad 000000+[0$ $000300+00 \quad 000000+C 0$ $000300+00 \quad 000000+60$ $000 \rightarrow 00+00 \quad 000000+00$ $000000+00 \quad 00000+0$ $000000+00$ 00000000 $000000+00 \quad 000000+00$ $000000+00$ 


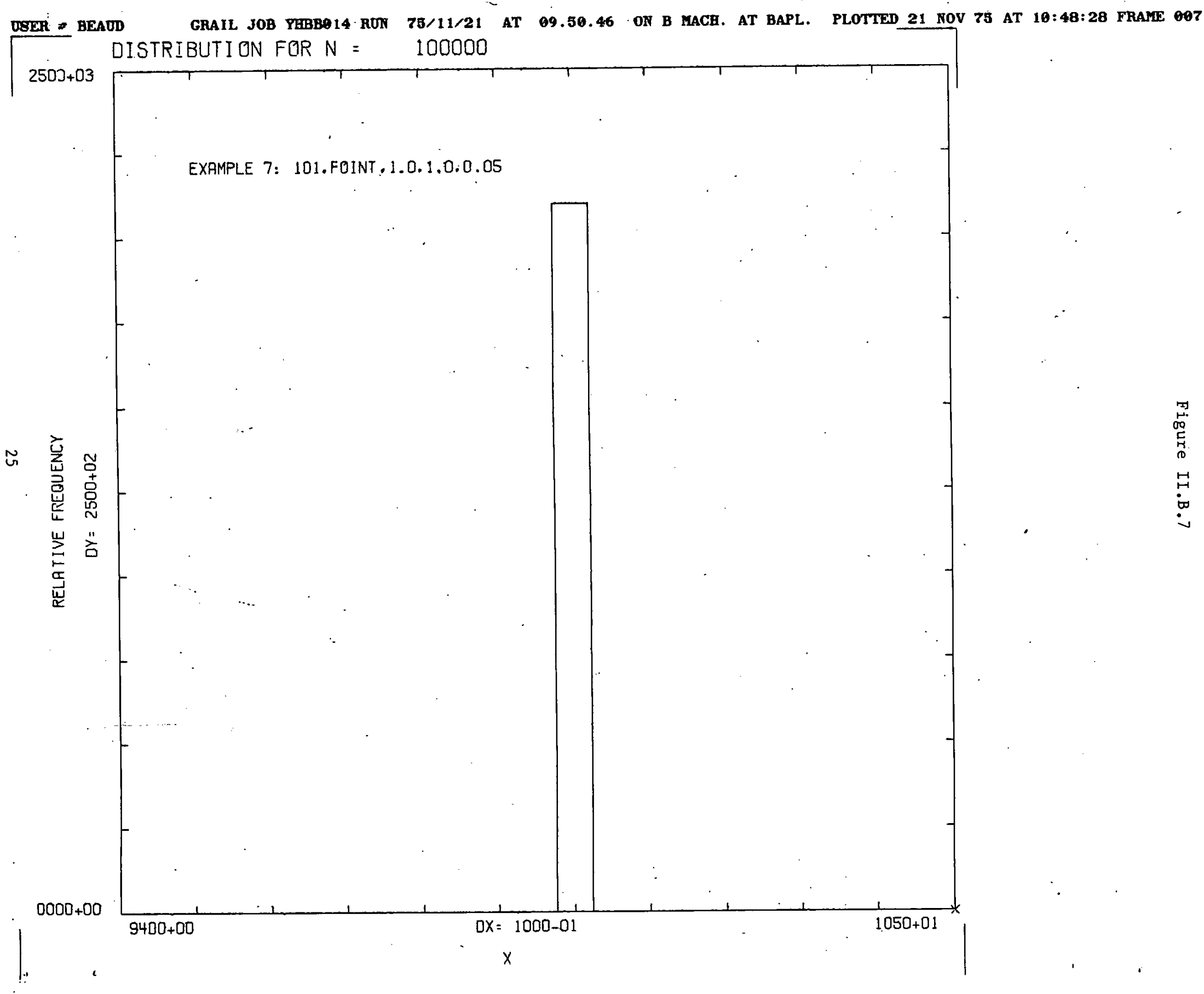


values in the range of 0.95 to 1.05 . Example 2 samples from a line distribution having a slope of 200.0 and a $y$-intercept of -189.9 . Example 3 samples from a normal distribution having a mean of 1.0 and a standard deviation of 0.0125 . Example 4 samples from a biased and truncated normal distribution having a mean of 3.025 and a standard deviation of 0.025 . Example 5 samples from a chisquare distribution which goes to 0 at 0.95 , peaks at 0.96 and has a degree of skewness (1) ) equald to 2. Fxample fi samples from a histogram 'distribution' having $10 \%$ of its population between 0.95 and $0.96333,30 \%$ between 0.93333 and 1.01666 and $40 \%$ between 1.01666 and 1.04999 . Example 7 tests the constant option for $x=1$. The results from these seven examples displayed in the referenced tables and figures demonstrate the use of METER's random number generator and verify that the random number eenerator performs as it was designed.

II. C. Use of METER to Program a Simulation

This section contains all of the detailed information needed to program and execute a METER Monte Carlo simulation. The instructions in II.C.I show how to construct a METER job input deck. Sections II.C.2 through II.C.4 present examples of the use of METER for fuel pellet volumes, fuel thermal conductivity, and tuel stack lengths.

II. ‥ 1. Dparational Procedure

The input deck for a METER job consists of three sections separated in such a way that each section becomes a record in the computer input f1le. The three sections or input records are: 
1. Control cards for retrleving required files, compiling the user's SIM routine, appending to MEIER, and executing METPR.

2. STM subroutine and any other FORTRAN subroutines or functions referenced in SIM and written by the user.

3. Input cards for executing the simulation.

The first record of input must contain the computer cards required to retrieve the METER program, the system's FORTRAN library, compiler, and loader; the cards required to compile the user's SIM subroutine and append it to METER; and the cards required to load and execute METER. These cards depend on the computer installation and will not be described in detail. The sample problems in section II.C show the control cards used at Bettis.

The second record of input must contain the user's SIM subroutine plus other routines as needed. Keypunching must start in column seven and the cards must be organized according to standard FORTRAN as follows:

SUBROUTINE SIM(XIN,XOUT)

DTEENSION XIN(I),XOUT(I)

COMMON/STM/NIN, NUU'1', MREPS, NUP, IVUUU'L, IAVAII (opt1OnaI)

Any number of FORTRAN statements

$\stackrel{-}{\bullet}$

RETURN

END

Other routines needed by SIM

:

End of record card

$$
27
$$


The XIN array contains the 1 or more (up to 99) independent random variables; the XOUT array contains the 1 or more (up to 99) dependent random variables. the FORTRAN statements mist define each XOUT( $i)$ as functions of the XIN( $j)$. During each repetition or history, METER will use its random number generator (II.A.2) and the distribution parameters obtained from the input cards ISS (II.A.I) to compute the XIN(j). METER will then call SIM to compute the XOUT(i).

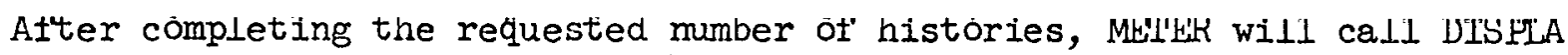
(II.A.3) to display the XOUT distributions.

Some useful information about the simulation in progress is stored in a labeled common block called SIM. The variables stored in the common block SIM are:

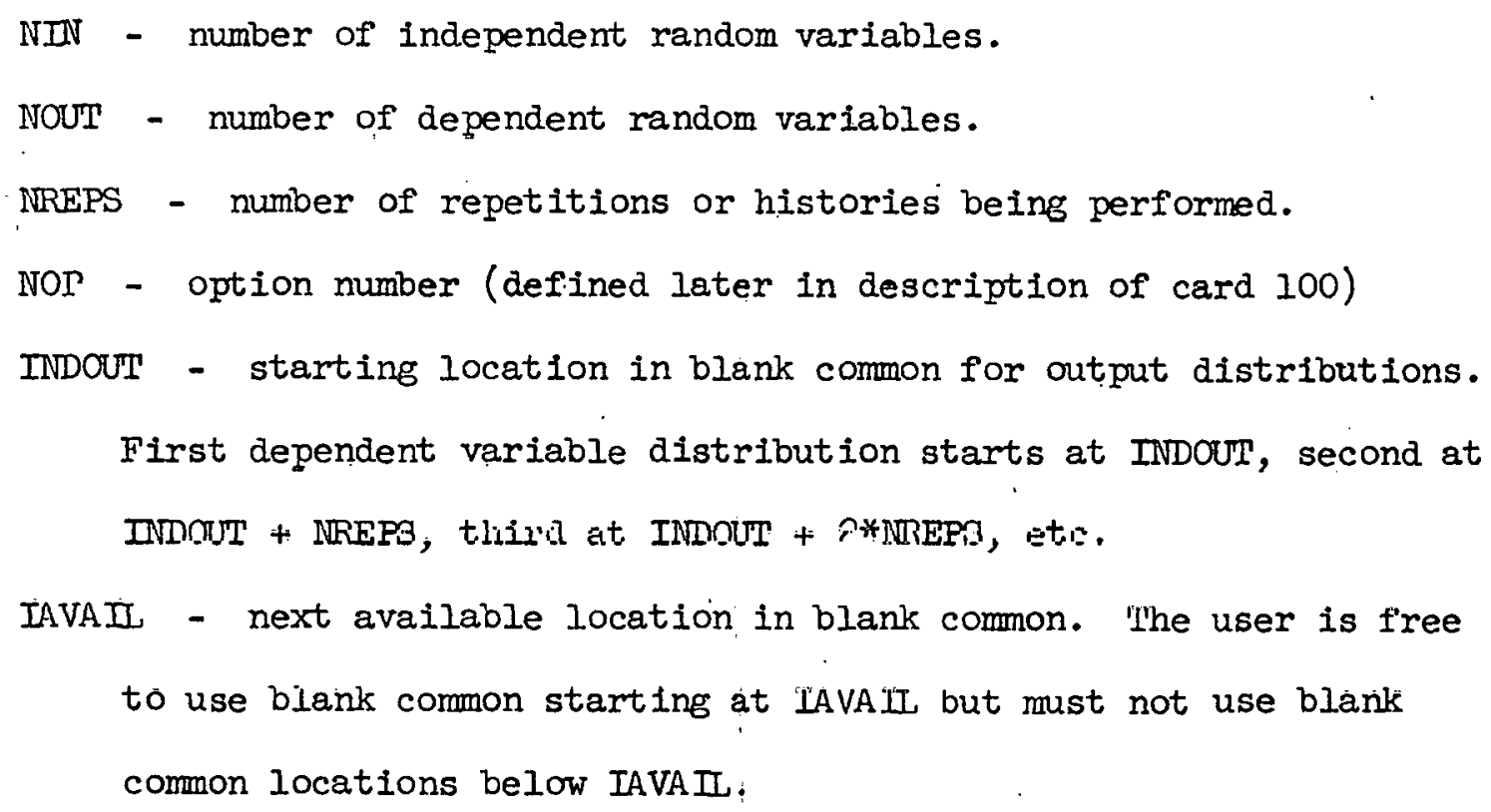

The above variables can be made available in the user's SDM subroutine by including the statement

$$
\text { COMMON/STM/NTN, NOUT, NREPS, NOP, INDOUT, IAVAII }
$$

at the front of the subroutine. 
The third record of the METER input deck must contain Bettis Input Package cards for controlling the execution of the simulation. These input cards are described below:

a. Title Card, optional: = followed by character string The Bett1s Input Package assumes that a card beginning with an equal sign $(=)$ is a title card. The character string on a title card will be printed at the top of each page of output.

b. Card 0, optional: 0, SIGMA, ICARRY, INTI

SIGMA = mumber of standard deviations at which to truncate all input (independent variable) normal distributions; default is 4.0 .

ICARRY $=I$ if input cards are to be carried over from case to case, 0 otherwise; default is 1.

INIT $=1$ if parameters of the input distributions are to be printed (debug aid), 0 otherwise; default is 0 .

c. Card 100, required: 100, ISTART, NIN, NOUT, NRE'PS, NOP ISTART $<0$ to generate the random numbers with a random start, 0 to use the standard start in RANDOM, $>0$ to begin the random numbers with a specified start based on ISTART.

$N I N=$ number of independent $\cdot$ random variables $(0<N I N \leq 99)$

NOUT $=$ number of dependent random variables to be displayed $(0 \leq$ NOUT $\leq 99)$. NREPS = number of repetitions or histories being performed. 
NOP $=1$ if the user's SIM subroutine simply defines the dependent variables; that is, random values for the independent variables are generated by METER, SIM computes the dependent variable values, METER displays results (normal use of METER). SIM is executed during each repetition.

NOP $=2$ if the user's STM will handle the entire simulation by Generating the independent random values uslue alrect calis on RANVAR, RV or a user supplied random number generator. SIM is called once. Output results wil.1 be displayed by METER using DISPLA if NOUT $>0$.

d. Cards 1ss, required: Iss, (DIST), XNOM, XTOL

The Iss cards are used to describe the frequency distributions to be used when generating the independent random variable values. A card is needed for each independent variable in the simulation. ss $=01,02,03, \ldots, N$ NIN.

Six types of distributions are allowed in MEIER; they are:

1. flat

2. Iine

3. normal

4. point

ว. chI square

6. histogram

Whenever an input distribution is requested by METER for a ra:idom variable, one of the above six distributions may be selected by specifying the distribution to be used and the parameters of that 
distribution. In the METER input caro description the term (DIST) is used to instruct the user to supply an option mumber or name and a list of parameters next on a card. The foll.owing describes what (DIST) means for the various distributions:

1. Flat Distribution

$$
\begin{aligned}
& (\text { DIST })=I_{I} \\
& I_{1}=1 \text { or FLAT. }
\end{aligned}
$$

2. Line Distribution

$$
\begin{aligned}
& (D I S T)=I_{1}, X_{1}, X_{2} \\
& I_{1}=2 \text { or IINE. } \\
& X_{1}=\text { slope of the line. } \\
& X_{2}=y \text { intercept of the line. }
\end{aligned}
$$

3. Normal Distribution

$$
\begin{aligned}
& (D I S T)=I_{1}, X_{1}, X_{2} \\
& I_{3}=3 \text { or N } \phi \mathrm{RMAL} . \\
& X_{1}=\text { mean of the normal distribution. } \\
& X_{2}=\text { standard deviation of the normal distribution. }
\end{aligned}
$$

4. Point Distribution (no randomness or variability)

$$
\begin{aligned}
& (\text { IIST })=I_{I}, X_{I} \\
& I_{I}=4 \text { or P } \phi \text { INT. } \\
& X_{1}=\text { value to be used. }
\end{aligned}
$$


5. Chi Square Distribution (skewed)

$(D I S T)=I_{1}, X_{1}, X_{2}, I_{2}$

$I_{1}=5$ or CHISQUARE.

$x_{1}=x_{0}-$ location where the value of the function is zero uppusite Irom the tail.

$x_{2}=x_{p}$ - location where the function peaks $\left(x_{1} \neq x_{2}\right)$. If $x_{1}$ $\left\langle\mathrm{x}_{2}\right.$, the tail of the distribution is to the right. If $\mathrm{x}_{1}$ $>X_{2}$, the tail of the distribution is to the lcft.

$I_{2}=$ the pmmeter $\nu(\geq 3)$ indicating the degree of skewness. The larger of the value of $v$, the more clusely the distribution approximates a normal distribution.

6. Histogram Distribution

$(D I S T)=. I_{1}, I_{2}, X_{1}, X_{2}, X_{3}, X_{4}, \ldots, X_{\ell}$

$I_{1}=6$ or HIST ØGRAM.

$I_{2}=$ the number of classes or steps in the histogram.

$x_{1}=$ left boundary of the first class.

$\dot{x}_{2}=$ width of each class.

$x_{3}$ through $x_{l}=y$ value for each cluss $\left(l=I_{2}+2\right)$.

XNOM = nominal value of the random variable.

XTOL $=$ tolerance on the random variable. Values of the variable will be taken from the specifled distribution over the range XNOM \pm XTOL.

e. Cards 2ss, optional: 2ss, (string)

This card series is used to supply titles for the tabular output frOm METER.

ss $=01,02,03, \ldots$, NOUT

string $=$ up to 60 characters (enclosed in parentheses) used by METER to title the tabular output for the $s s^{\text {th }}$ dependent variable distribution. 
f. Caras 3ss, optional: 3ss, (string)

This card series is used to supply titles for the graphical outprit from METER.

ss $=01,02,03, \ldots$, NOTT

string $=$ up to 30 characters (enclosed in parentheses) used by MEIER to title the Eraphical output for the ssth dependent variable distribution.

B. Cards 4ss, optional: 4ss, (string)

This card series is used to supply the units of the dependent varlables for the tabular and graphical output. ss $=01,02,03, \ldots$, NOUT string $=$ up to 10 characters ised by MEIER to label the units of the ss $^{\text {th }}$ dependent variable.

h. Cards 5ss, optional: 5ss, X

This card series is used to control the histograms generated by METER. If not supplied, each output histogram will contain 19 classes or steps.

ss $=01,02,03, \ldots$, îH

$X=$ if fixed point, the number of classes or steps in the histogram for the $s s^{\text {th }}$ dependent variable; if floating point, $X$ is used as the sitep size. In either case, the maximum number of classes allowed is 101 . 


\section{i. End Case Terminator, required}

Each case of input (except the last) must be terminated by a card with a slash (/) as the first non-blank character.

\section{j. End Input Terminator, required}

The last case of input must be terminated by a card with a period (.) as the first non-blank character.

The next three sectione of this report will present examples of MEIFR input and output. All input values in the sample projlems were chosen strictly for the murpose of illustration.

II. C. 2. Sample Problem 1

For the first example, we will simulate the distributions of fuel pell.et cross sectional area and volume given the distributions for pellet diameter and length. We will ask METER to display the distributions for diameter, length, area, and volume. (Even though diameter and length are inclependent variables in this simulation, they can be displayed as dependent variables.

Page 37 contains a listing of the input deck for Sample.Problem 1, showine the three records of input: (a) the control cards, (b) the SIM subroutine, and (c) the variable input. The control cards will depend on the instaliation (the Bettis control cards are shown). The SIM subrout ine has two incoming variables $X I N(1)$ and $X I N(2)$ which are diameter and length respectively and returns four computed variables XouT(I), XOUI'(2), XOUT(3), and $\operatorname{XOUT}(4)$ which are diameter, length, area, and volume respectively. During execution, the values in the $X I N$ array will be 
supplied by METER's random number generator for each repetition. SDM will compute the values for the XOUT array and return them to METIR to be displayed at the end of the job.

The third record of input contains one case of input for the simulation: Card 100 requests the following:
a. The standard start (ISTART=0)
b. 2 input variables $(\mathrm{N} I N=2)$
c. 4. output variables (NOUT=4)
d. 1,000 histories or repetitions (NREPS=1000)
e. The standard METER run (NOP=1)

Card 101 defines the distribution for pellet diameter (card Iss is for $X I N(s s))$ to be a normal distribution with a mean of .252 and a standard deviation of .00012 . The specified range on diameter is $.252 \pm .0005$. Note that the requested range is $\pm 4.25060005 \% .00012)$; however, this will be truncated to $\pm 4 \sigma$ or $.252 \pm .00048$ because card 0 was not input to override the built-in $\pm 4 \sigma$ truncation. Card 102 specifies the distribution of pellet length to be normal with mean .615 and standard deviation .00165 over the range $.615 \pm .02$ which will be changed to $.615 \pm .0066$ by the $4 \sigma$ truncation. The 200, 300, and 400 series of cards supply the appropriate labels for the output distribution.

Pages 38 through 46 contain the output from the METER mun showing the histograms and statistics for the output distributions. Note that the actual pellet diameters and lengths fell within the requested ranges. Since the diameter and length distributions were specified to be unbiased 
(mean = nominal on cards 101 and 102), the means of the dependent variable distributions (area and volume) are close to the nominal values. This would not be the case had biased or skewed distributions been specified. The novice user might find it beneficial to experiment with this simple simulation of pellet area and volume by running several cases varying the input distributions. 
YHBBO0 1, 6, $001,140,1546$. MFTER, BEAUD, U, P . MIERGE( I = PIETER)

CCREAD ( METERCC, PLOTER=GRAVER)

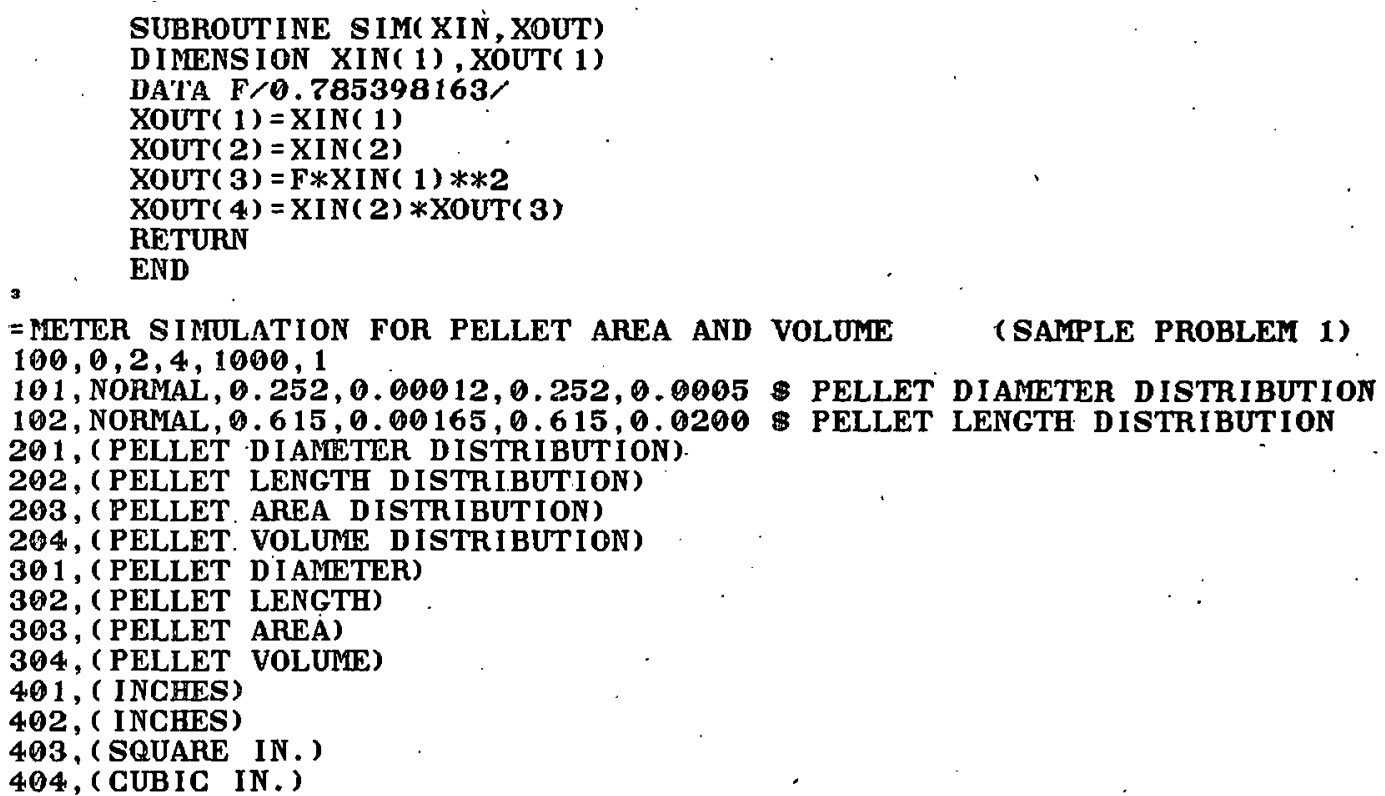



= METER SIMULATION FOR FELEET AREA AND VOLUNE (SAMPLE PROBLEM 1)

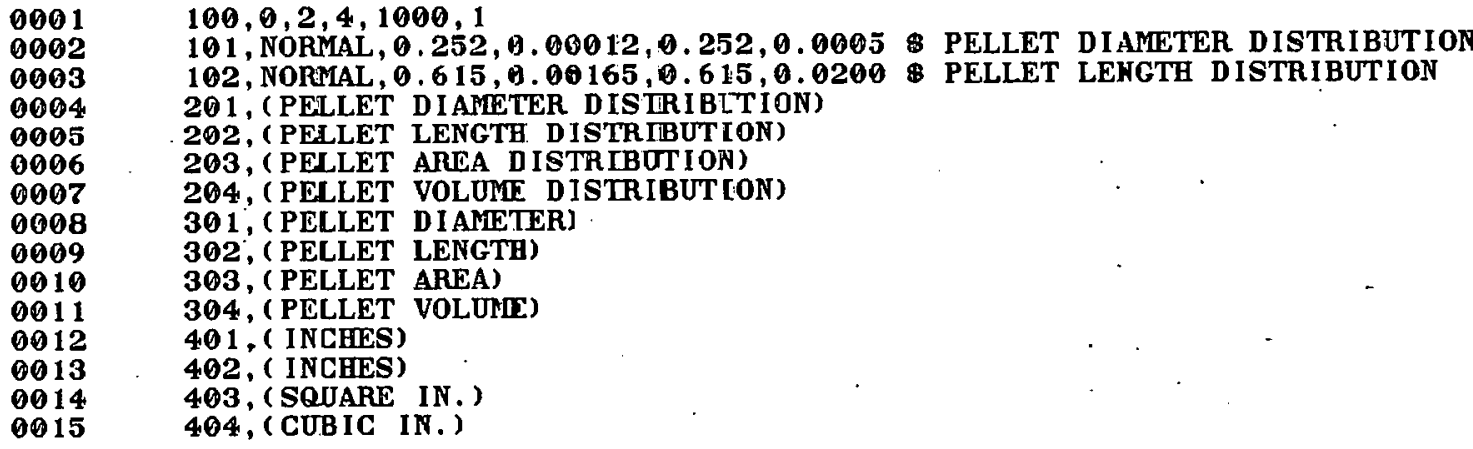
$100,0,2,4,1000,1$

0002

0003

0004

0005

0006

0007

0008

0009

0010

0011

0012

0013

0014

0015

101, NORMAL, $0.252,0.00012,0.252,0.0005 \%$ PELLET DIAMETER DISTRIBUTION

102, NORMAL, $0.615,0.00165,0.615,0.0200$ PELLET LENGTH DISTRIBUTION

201, ( PELLET D I AFETER D ISTRIBLTION)

202, ( PELLET LENGTH DISTRIBUTLON)

203, ( PELLET AREA DISTRIBUTION)

204. (PELLET VOLUMT DISTRIBUTLON)

301 , ( PELLET DIAMETER)

302, ( PELLET LENGTH)

303. (PELLET AREA)

304, ( PELLET YOLUNE)

401 , ( INCHES)

402. (INCHes)

403. ( SQUARE IN.)

404, ( CUB IC IN.)

KEY VARI ABLES

$\begin{array}{rrr}\text { SIGMA } & = & 4.0000 \\ \text { S } & 0 \\ \text { ICARRY } & = & 0 \\ \text { INIT } & = & 0 \\ \text { INRAN } & = & 1525878906250 \\ \text { NCASE } & = & -1 \\ \text { NDATA } & = & 70 \\ \text { CLASES } & = & i 9 \\ \text { IND1 } & = & 71 \\ \text { IND2 } & = & 73 \\ \text { IND3 } & = & 77 \\ \text { IND4 } & = & 161 \\ \text { IND5 } & = & 165 \\ \text { IND6 } & = & 4165 \\ \text { IAVAIL } & = & 4163 \\ \text { AMOUNT } & = & 31954 \\ \text { IT } & = & \text { OUTPUT } \\ \text { NER } & = & 0 \\ \text { DISPAR } & = & 112 \\ \text { NIN } & = & 2 \\ \text { NOUT } & = & 4 \\ \text { NREPS } & = & 1000 \\ \text { NOP }= & 1\end{array}$


CASE 1

PELLET DIAMETER DISTRIBUTION

$\begin{array}{cc}\text { CLASS } & \begin{array}{c}\text { (UNITS ARE } \\ \text { MINIMUM }\end{array} \\ 1 & 251628+00 \\ 2 & 251669+00 \\ 3 & 251710+00 \\ 4 & 251750+00 \\ 5 & 251791+00 \\ 6 & 251832+00 \\ 7 & 251873+00 \\ 8 & 251914+06 \\ 9 & 25195400 \\ 10 & 25199+00 \\ 11 & 252036+00 \\ 12 & 252077+00 \\ 13 & 252117+00 \\ 14 & 252158+00 \\ 15 & 252199+00 \\ 16 & 252240+00 \\ 17 & 252281+00 \\ 18 & 252321+00 \\ 19 & 252362+00 \\ & \end{array}$

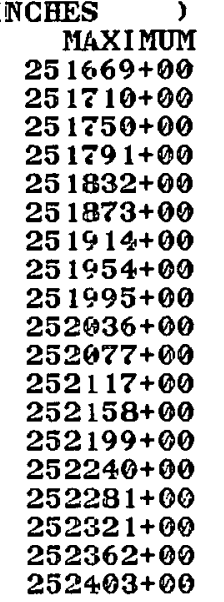

Courts
6
2
6
22
99
59
99
90
195
140
153
130
90
75
31
26
17
6
1
2

$P(x)$

600000-02

200000-02

$600600-02$

$30000-01$

39001

$59000-01$

$90000-01$

$105000+00$

$140000+00$

$153660+00$

$130000+00$

$900000-01$

$750030-01$

$310000-01$

$260000-01$

$170000-01$

$600000-02$

$100000-02$
$200000-02$

$\mathbb{N}=\quad 1000$

MINIMUI $=251628+00$

MAXIMUUI $=252403+00$

MEAN $=252001+00$

VAR IANCE $=137087-07$

STAYDARD DEVIATION $=117.084-03$

COEFF ICIENT OF SKEWNESS $=-292544-02$

$* * * * * * * * * * *$ NOMINAL VALUE $=252000+00$ 
METER SIMULATION FOR PELLET ARFA AND VOEUME

(SAMPLE PROBLEM 1) CASE 1

PELLET LENGTH DISTRIBUTION

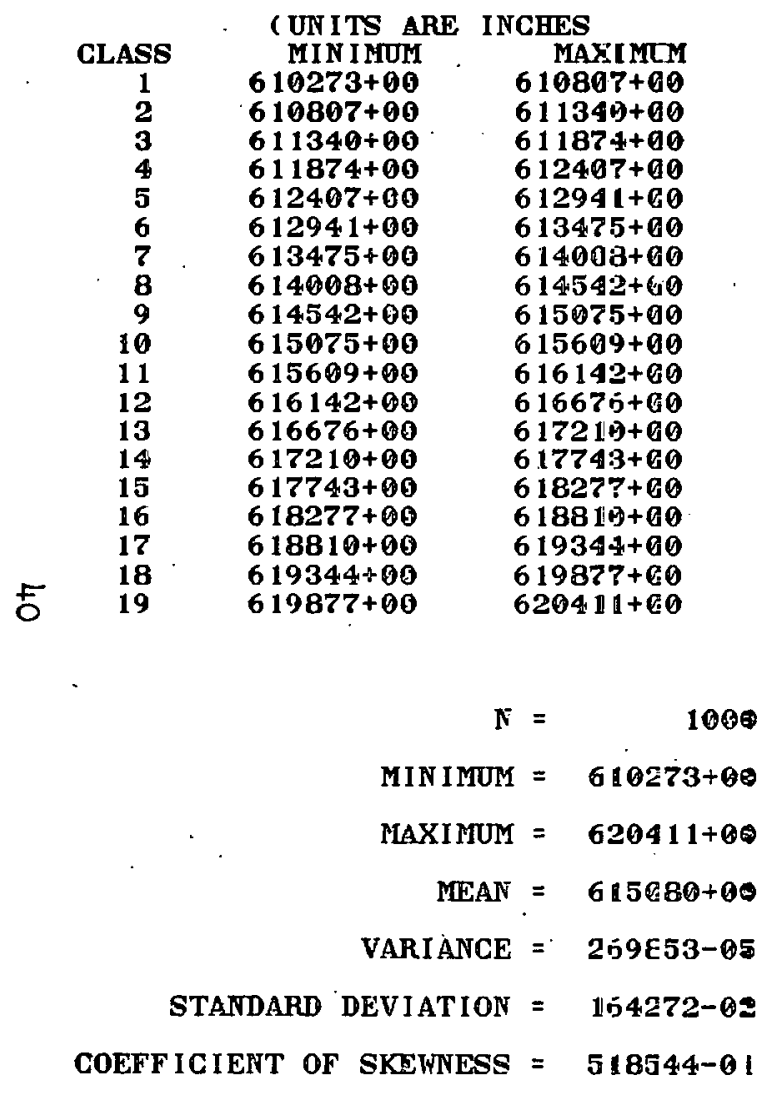

$* * * * * * * * * *$ NOMINAL VALUE $=515000+00$ 
CASE 1

PELLET AREA DISTRIBUTION

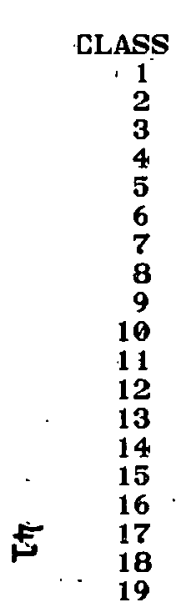

$\begin{array}{cc}\text { (UNITS ARE SQUARE IN.) } \\ \text { MIN IMUM } & \text { MAXIMUM } \\ 497288-01 & 497450-01 \\ 497450-01 & 497611-01 \\ 497611-01 & 497773-01 \\ 497773-01 & 497934-01 \\ 497934-01 & 498095-01 \\ 498095-01 & 498257-01 \\ 498257-01 & 498418-01 \\ 498418-01 & 498580-01 \\ 498580-01 & 498741-01 \\ 498741-01 & 498903-01 \\ 498903-01 & 499064-01 \\ 499664-01 & 499255-01 \\ 499225-01 & 499387-01 \\ 499387-01 & 499548-01 \\ 499548-01 & 499710-01 \\ 499710-01 & 499871-01 \\ 499871-01 & 506032-01 \\ 500032-01 & 509194-01 \\ 500194-01 & 506355-01\end{array}$

$\begin{array}{rc}\text { CoUTTS } & P(X) \\ 6 & 600000-02 \\ 2 & 200000-02 \\ 6 & 600000-02 \\ 22 & 220000-01 \\ 40 & 400000-01 \\ 58 & 580000-01 \\ 90 & 900000-01 \\ 108 & 108000+00 \\ 137 & 137000+00 \\ 153 & 153000+00 \\ 131 & 131000+00 \\ 90 & 900000-01 \\ 74 & 740000-01 \\ 32 & 320000-01 \\ 25 & 250000-01 \\ 17 & 170000-01 \\ 6 & 600000-02 \\ 1 . & 100000-02 \\ 2 & 200000-02\end{array}$

$$
\begin{array}{rr}
\text { N } & =1000 \\
\text { MIN IMUM } & =497288-01 \\
\text { MAXIMUI } & =500355-01 \\
\text { MEAN }= & 498764-01 \\
\text { VARIANCE } & =214803-08
\end{array}
$$

STAIVDARD DEVIATION $=463468-04$

COEFF IC IENT OF SKEWNESS $=-138123-02$

$* * * * * * * * * *$ NOMINAL VALUE $=498759-01$ 
METER SIMULATION FOR PELLET AFEA AND VDLUME

CASE 1

PELLET VOLUME DISTRIBUTION

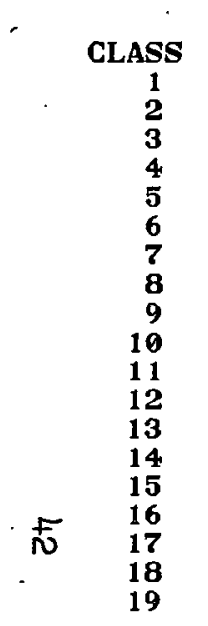

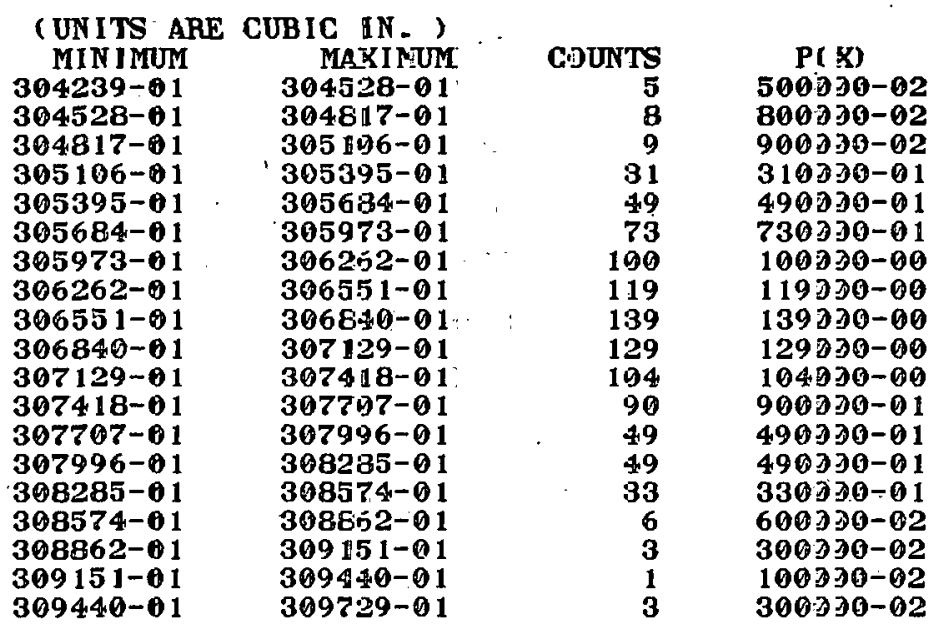

$\mathbf{N}=$

1000

MINIMUM $=304239-01$

MAXIMUM $=309729-01$

MEAN $=306780-01$

VARI ANCE $=763502-08$

STANDARD DEVIATION = 873785-64

COEFF ICIENT OF SEEWNESS $=\cdot 59665-01$

*********** NOMINAL VALUE $=3 G 6737-01$

IINAN $=2651046790$ 
USER - BEAUD METER JOB YHBB (040) RUN 76/10/08 AT 08.42.23 ON A MACH. AT BAPL. PLOTTED B OCT 76 AT 8:46: 12 FRAME O01

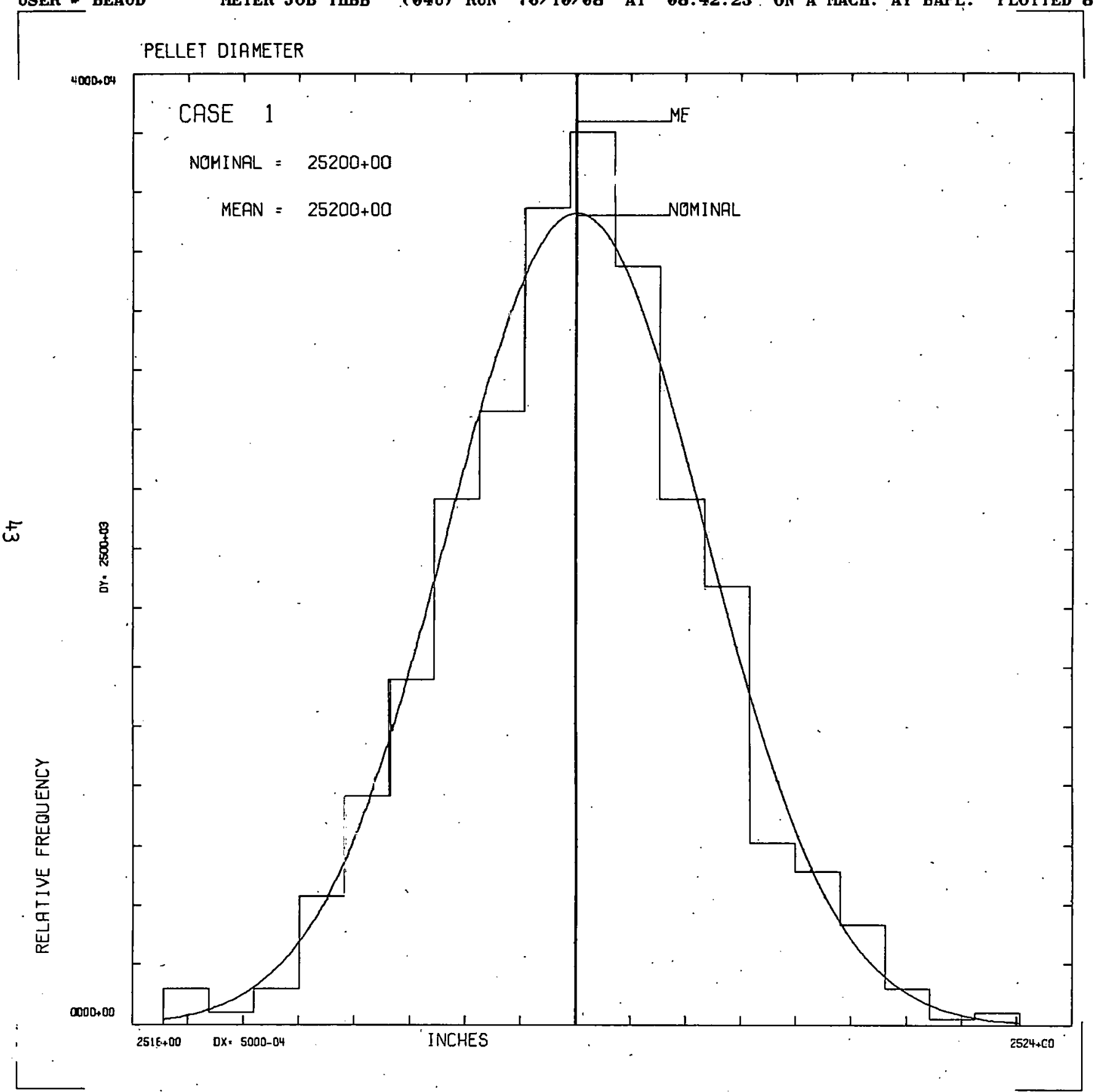


USER * BEAUD

METER JOB YHEB (04U) RUN $76 / 10 / 08$ AT 08.42.23 ON A MACB. AT BAPL. PLOTTED 8 OLT 76 AT B:47:46 FRAIE 002

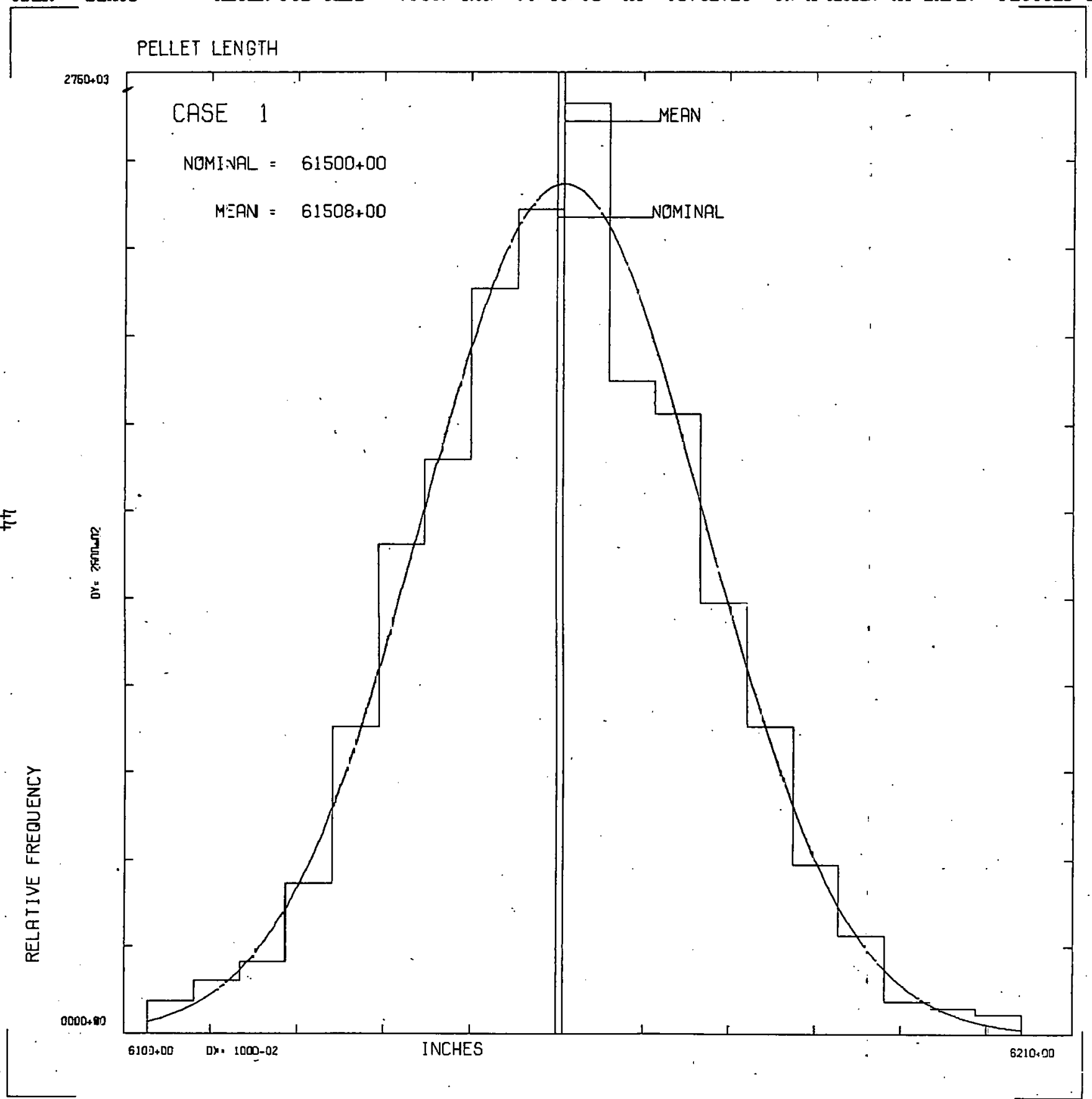


UQER - BEAUD TETER JOB YHBB (04U) RUN 76/10/08 AT 08,42.23 ON A MACH. AT BAPL. PLOTTED a OCT 76 AT B:49:24 FRAME 003

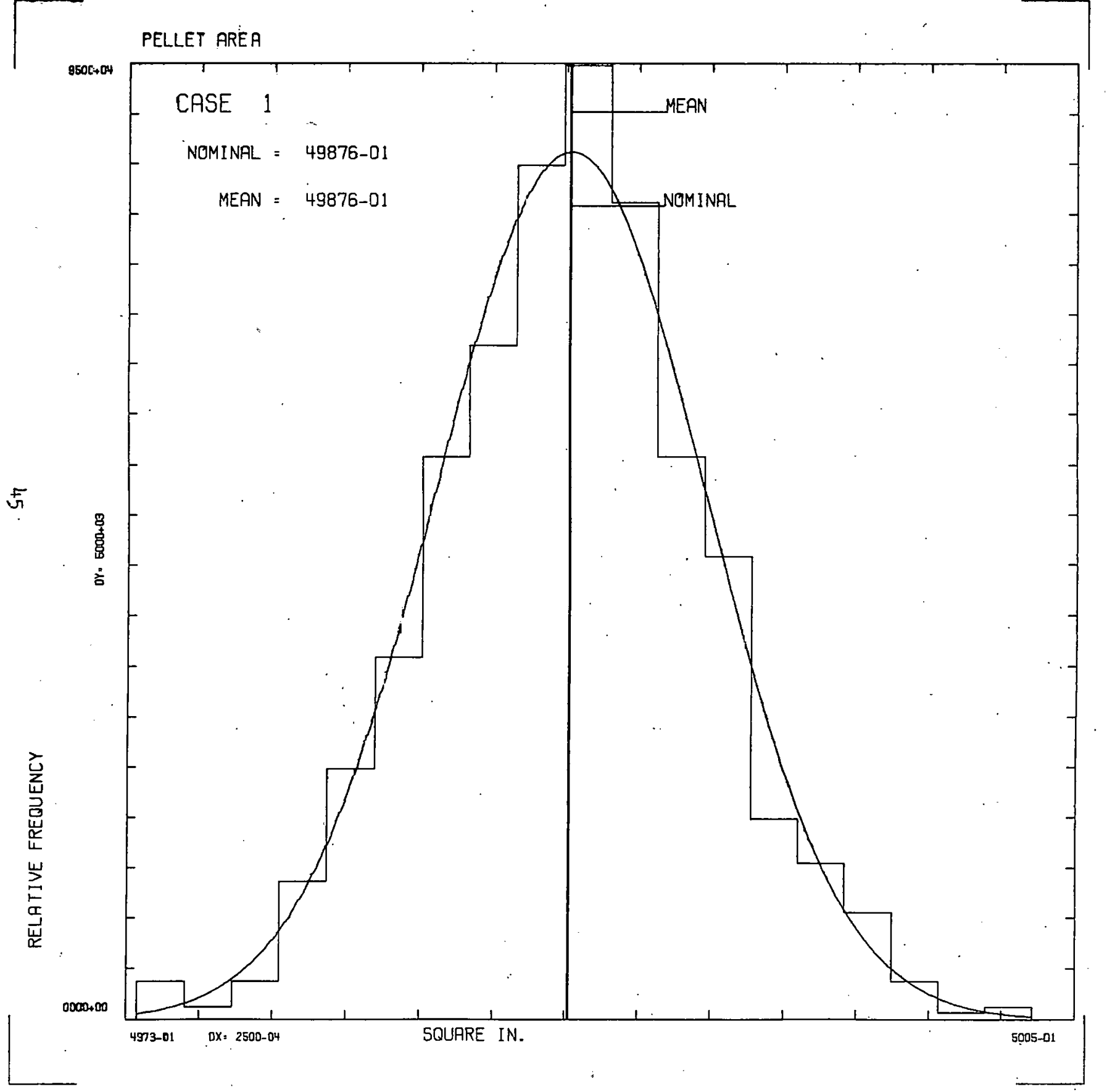


USER - BBAUD METER JOB YHBB (O4U) RUN 76.10/08 AT 08.42.23. OH A MACH. AT BAPL. PLOTTED 8 OCT 76 AT B:51:28 FRAME 004

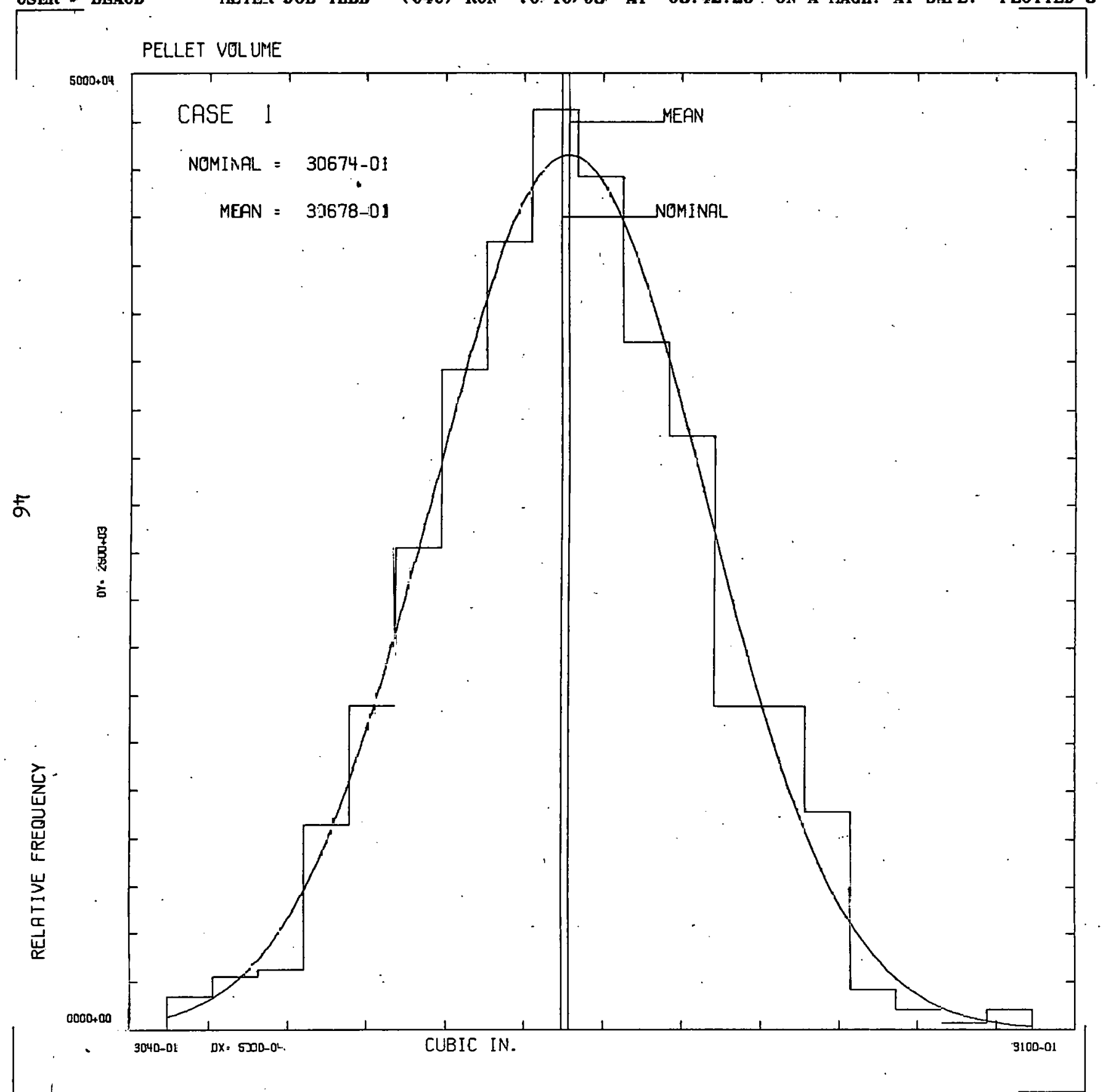




\section{C. 3. Sample Problem ?}

The second sample problem dea].s with fuel thermal conductivity. Suppose we have a model which predicts fuel thermal conductivity - $K$ in units of $B T U / \mathrm{Hr}-\mathrm{Ft}-{ }^{\circ} \mathrm{F}$ as a function of fuel fractional porosity - $P$, fuel depletion - $F$ in units of $10^{20}$ fissions/cc, fuel temperature - $T$ in degrees Kelvin, and weight fraction $\mathrm{UO}_{2}-U$ as follows:

$$
K=\alpha \frac{1-P}{1+\beta P}\left[A_{0}+A_{1} U+A_{2} U^{2}+\left(B_{0}+B_{1} U+B_{2} U^{2}\right) T+\frac{C+\text { IF }}{T}\right]^{-1}
$$

where:

$$
\begin{aligned}
& \alpha=57.78 \\
& \beta=1.15 \\
& c=1,800 \\
& D=616
\end{aligned}
$$

We wish to evaluate fuel thermal conductivity under various conditions Given estimates of the coefficients $A_{1}$ and $B_{i}$ and given uncertainties in the estimates. The input for such a Monte Carlo calculation is shown on page 49. The SIM subroutine assumes six input random variables $X(1)$ through $X(6)$ which represent $A_{0}, A_{1}, A_{2}, B_{0}, B_{1}$, and $B_{2}$ respectively. The I1sted problem assumes a fuel porosity of 0.03 , a fuel depletion of 0 (beginning of life), a fuel temperature of $1000^{\circ} \mathrm{K}$ and a $\mathrm{UO}_{2}$ welght fraction of 0.05 . These four variables are treated as constants whose values are assigned by FORTRAN "DATA" statements. Card 0 is supplied in the input to change the normal distribution truncation from $\pm 4 \sigma$ to $\pm 6 \sigma$. Card 100 requests a random start, six input or independent random variables, one output or dependent random variable, 10,000 repetitions 
and option 1. Distributions for the coefficients are defined on cards 101 through 106. Cards 201, 301, and 401 supply output labels and card 501 requests that the output histogram be generated with a step size of 0.05

The output from sample problem 2 is presented on pages 50 through 52 The mean thermal conductivity for 10,000 repetitions was 2.33191 ; the expected value based on the nominal values on cards 101 through 106 was 2.32942. The uncertainty in fuel thernal conductivity predicted by METER for the sample problem is 0.0920 at the $1 \sigma$ level. 
YHBB002, 6,001, 140, 1546. METER, BEAUD, U, $P$. MERCE $(I=$ METER)

CCREAD (METERCC, PLOTER= GRAVER)

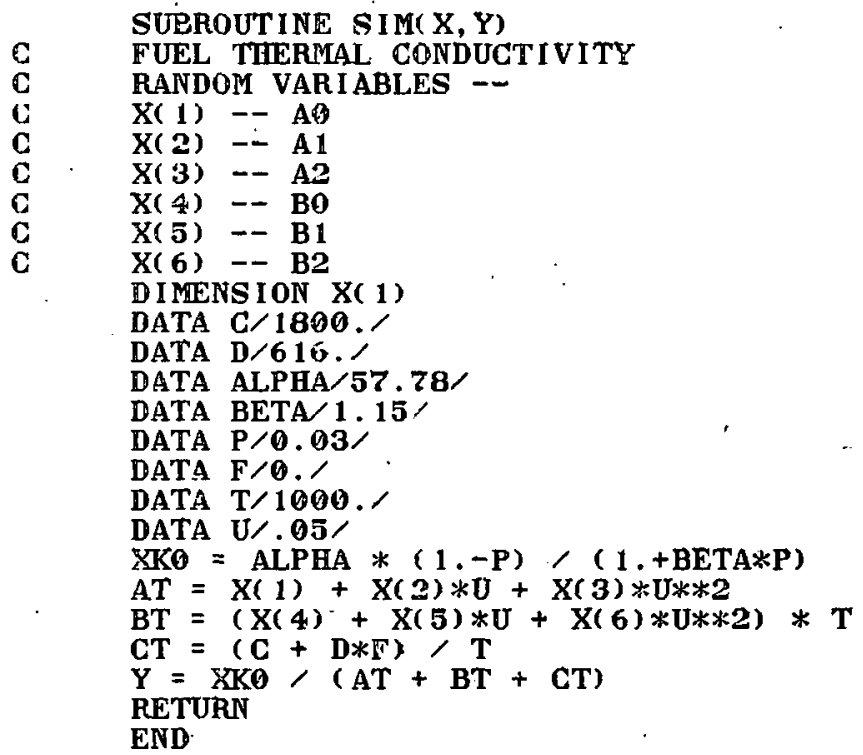

= METER SIMULATION FOR FUEL THERMAL CONDUCTIVITY $0,6.0,1,0$

$100,-1,6,1,10000,1$

101 , NORMÁL, $0.931370,0.046568,0.931370, .3$

102, NORMAL, 20.83000; $1.041500,20.83000,7$.

103, NORMAL, -281.018, 14.05090,-281.018,85.

104 , NORMAT, O.018220,0.000911,0.018220,.006

105 , NORMAL, 0.034136, $0.001707,0.034136, .011$

106 , NORMAL, 0. 104320, .0052 16,0.104320; .032

$\$$ COEFFICIENT AO

$\$$ COEFF ICIENT A1

\& COEFF ICIENT. A2

S COEFFICIENT BO

S CUEFFICIENT. B 1

201 , ( FUEL THERMAL CONDUCTIVITY AT $P=0.03 \quad F=0 \quad T=1000 \mathrm{~K} U=50 / 0$ )

301 , ( FUEL THERMAL CONDUCTIVITY)

$401,($ BTU/HIFTOF)

$501,0.05$ 
LISTING OF' INPUT DATA FOR GASE 1 = HETER SIMULATION FOR FUEL THERMAL CONDUCTIVITY

(SAMPLE PROBLEM 2)

0001
0002
0003
0004
0005
0006
0007
0008
0009
0010
0011
0012

$$
\begin{aligned}
& 0,6.0,1,0 \\
& 100,-1,6,1,10000,1
\end{aligned}
$$

101 , NORMAL, $0.931370,0.046568,0.93: 370, .3$ 's COEFFICIENT $A 0$ 102, NORMAL, $20.83000,1.041500,20,33000,7 . \quad$ S COEFFICIENT A1 103, NORMAL, $-281.018,14.05600,-281.018,85.98$ COEFFICIENT A2 104, NOFMAL, $0.018220 .0 .0009 \mathrm{1}, 0.018220, .006$ S COEFFICIENT BO 105, NOFIAL, 0.034136.0.001767,0.03136,.011 \$ COEFFICIENT B1 106, NORMAL, 0. 104320.0.005216,0.134320,.032 \$ COEFFIC1ENT B2 201, $($ FUEL THERUA CONDUCTI ITY

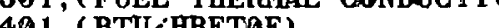
, ( BTO HR $501,0.05$

\begin{tabular}{|c|c|}
\hline S IGMA & 6.0000 \\
\hline ICARRY & 1 \\
\hline IN IT & 0 \\
\hline INRANT & $=$ \\
\hline NCASE & -1 \\
\hline NDATA & 74 \\
\hline CLASES & 191 \\
\hline I ND 1 & 75 \\
\hline IND2 & 81 \\
\hline IND3 & 82 \\
\hline IND4 & 154 \\
\hline IND5 & 155 \\
\hline IND6 & 10155 \\
\hline IAVAIL & 10459 \\
\hline AMOUNT. & 31909 \\
\hline $\mathrm{IT}$ & $=$ OUTPUT \\
\hline NER & $=$ \\
\hline DISPAR & 12 \\
\hline NIN & 6 \\
\hline NouT & 1 \\
\hline NREPS & 10000 \\
\hline NOP & 1 \\
\hline
\end{tabular}

\section{KEY VARIABLES}


CASE 1

FUEL THERMAL CONDUCTIVITY AT $P=0.03$

$F=0 \quad T=1000 K \quad U=5 \quad 0 / 0$

CLASS
1
2
3
4
4
5
6
7
8
9
10
11
12
13
14
15

$\begin{array}{cc}\text { (UNITS ARE } & \text { BTU/HRFTOF) } \\ \text { MINIMUM } & \text { MAXIMUM } \\ 200000+01 & 205000+01 \\ 205000+01 & 210000+01 \\ 210000+01 & 215000+01 \\ 215000+01 & 220000+01 \\ 220000+01 & 225000+01 \\ 225000+01 & 230000+01 \\ 230000+01 & 235000+01 \\ 235000+01 & 240000+01 \\ 240000+01 & 245000+01 \\ 245000+01 & 250000+01 \\ 250000+01 & 255000+01 \\ 255000+01 & 260000+01 \\ 260000+01 & 265000+01 \\ 255000+01 & 220000+01 \\ 270000+01 & 275000+01\end{array}$

COUNTS
1
26
134
599
1230
1922
2196
1812
1194
670
276
82
28
7
3

$P(X)$

100000-03

260000-02

134000-0

$509000-01$

$123000+00$

$192200+00$

$210600+00$

$181200+00$

$119400+00$

$670000-0$

276000-01

820000-02

280000-02

700000-03

300000-03

$N=10000$

MINIMUM $=203855+01$

MAXIMUM $=272373+01$

MEAN $=233191+01$

VARI ANCE $=$ 846935-02

STAINDARD DEVIATION = 920291-01

COEFF IC IENT OF SKEWTESS $=245121+00$

$\approx * * * * * * * * * *$ NOMINAL VALUE $=232942+01$

INRAN = 2017058874 


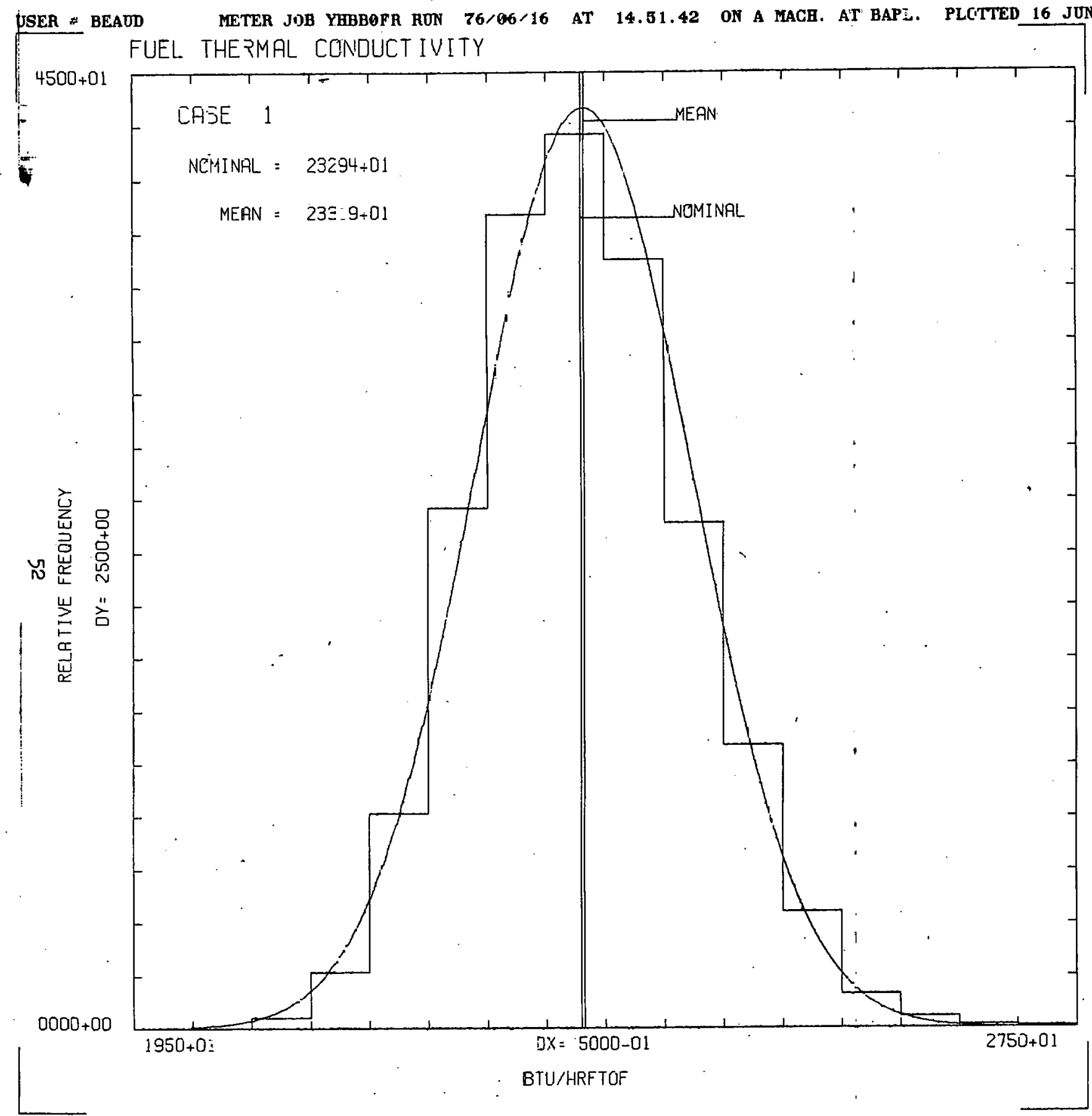


II. C. 4. Sample Problem 3

The third sample problem uses METER to simulate a relatively simple manufacturing process--stacking fuel. pellets of random length to form fuel. stacks of specified tolerances. This simulation utilizes option 2 (see description of card 100 in II.C.I) because the entire simulation must be programmed in the SIM subroutine. The coding requires more FORTRAN proficiency than the, two prior examples and the author has chosen to use the Bettis Input Package to process an additional card of input rather than set certain variables directly vithin the SIM subroutine.

The steps in the simulation are as follows:

a. determine minimum and maximum c.jowable stack lengths.

b. since peliet lengt? distributions and the stack length requirements could conflict and make it difficult to complete stacks; determine the maximum attempts at completing a stack and set the failure counter to 0 .

c. initialize a new stack by setting its try counter to 0 and its length to 0 .

a. select a random pellet for the stack; its length will be a random variable.

e. add the new pellet's length to the length of the stack.

r. repeat (d) and (e) until the stack length equals or is greater than the whiluum required stack length determined at step (a). 
g. if the stack length is less than or equal to the maximum required stack length, save its value for the output distribution and return to step (c). If the desired number of stacks have been produced, terminate the simulation and display the results.

h. If the stack length is greater than the maximum required length, up the try counter by 1 . If the try counter is greater than the maximum attemptis set at step (b), up the failure counter by 1 , discard this stack and return to step (c).

1. if allowed to try again, remove the last pellet (subtract its length from the stack length) and return to step (d) in hope of finding a shorter pellet.

The input deck for sample problem 3 is listed on pages 56 and 57 Each FORTRAN statement in SIM is preceeded by a comment which explains that statement. Note that when option ' 2 of' MEIER is used (NOP $=2$ ), SIM must bc prepared for 2 passes--pass 1 to return nominal values for all dependent random variables (second argument) and pass 2 to perform the simulation. Pass 1 for our sample problem has been programmed to read card 1 to obtain the nominal stack length, the stack length tolirance and the maximum number of attempts at finishing a stack. Since card 1 in the example contains $84.0,0.368$, and 5 respectively for the constants, the pass I coding coulá huve luen

$$
\begin{aligned}
& \text { SLNOM=84. } \\
& \text { SLTOL=. } 368 \\
& \text { NIRIES }=5 \\
& \text { Y=SLNOM } \\
& \text { NPASS=2 } \\
& \text { RETURN }
\end{aligned}
$$


The constants could have been changed within STM for each run. The advantages in coding SIM the author's way are (a) the variable input is centralized in record 3 (card input) and (b) the user's own version of METER can be saved on Iine for the duration of his application without further need to compile and recompile SIM during each run. At Bettis this would be accomplished by including

PROGM(FM)

$\operatorname{STORE}(I=$ MEIER, $T=62, \quad \mathrm{~V}=\mathrm{nnn}, \mathrm{F}=\mathrm{S}, \mathrm{S}=\mathrm{METER})$

(where $1 \leq n n n \leq 999$ ) after the MERGE card and by including

$$
\text { FM(STORE, METER) }
$$

after the CCREAD card.

Subsequent runs would require the following simplified input deck

Job Card

FETCH( $I=M E T E R, T=62, V=n n n, F=S, S=M E T E R$ )

METER.

end record

Bettis Input Package Cards.

The output results from sample problem 3 are shown on pages 58 through 60 Note that no stack falled to be completed but that the resulting stack length distribution is somewhat peculiar. No stacks were "made" by MEILEK with the nominal stack length and we observe 2 peaks in the distribution. The combination of the stack length requirements $(84.0 \pm 0.368)$ and the pellet length distribution $(\eta=0.615, \sigma=0.002$, between $0.615 \pm 0.020$ produces stacks containing either 136 or 137 pellets. It must be emphasized that this simulation does not describe all aspects of an actual stacking operation and was simplified for the purpose of demonstrating the use of METER. 
YHBBO03, 6, $001,140,1546$. METER, BEAUD, U, P.

MERGE ( I = METER)

CCREAD ( METERCC , PLOTER= GRAVER)

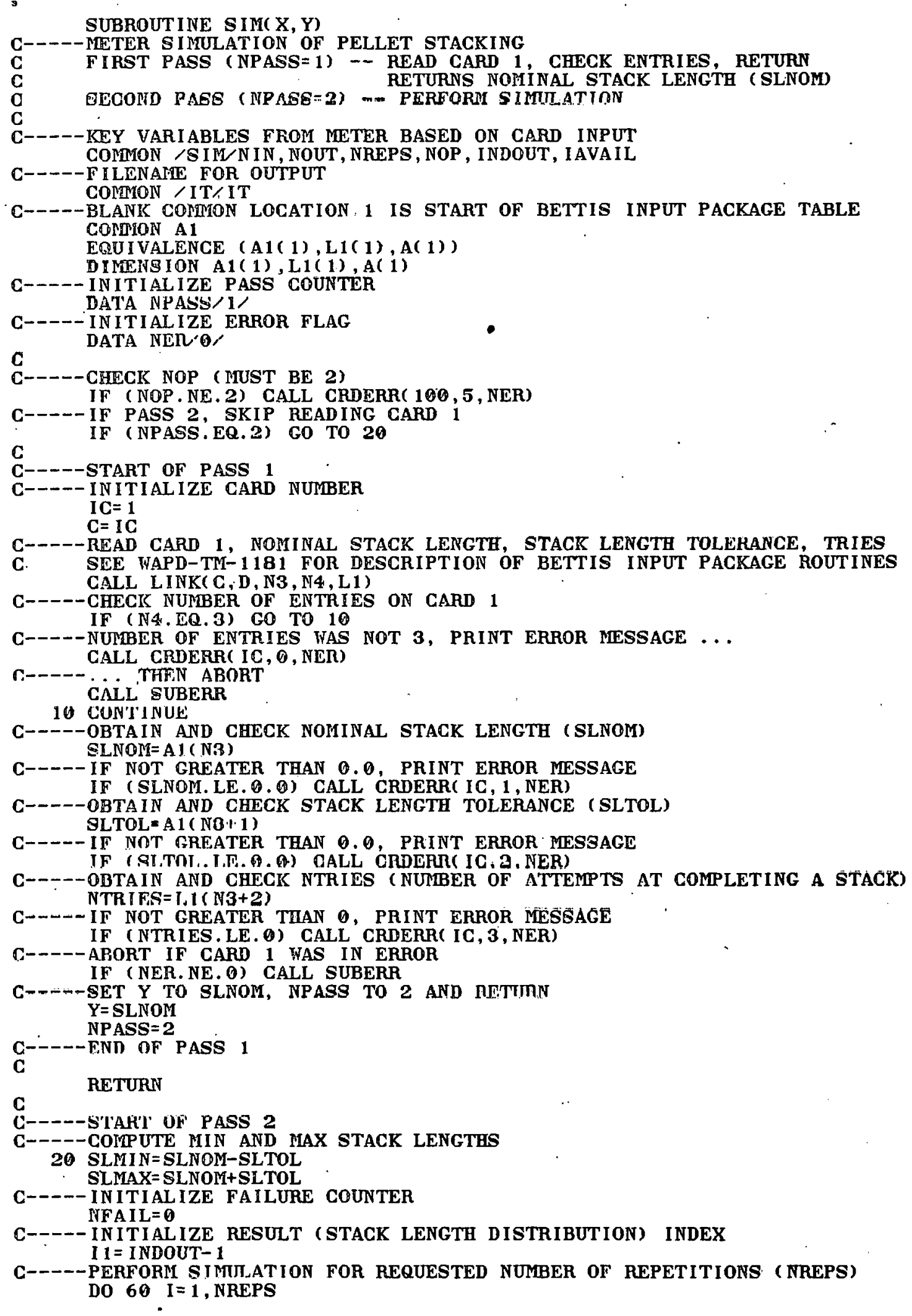


C-----INCREMENT RESULT INDEX BY 1 $I 1=I 1+1$

C-D- INITIALIZE COUNTER ON NURBER OF ATTEMPTS TO COPIPLTE THE STACK 30 NTRY =

C-----INITIALIZE STACK LENGTH TO 0.0 (NEW STACK) $S L=0$.

C----OBTA IN NEXT RANDOM PELLET LENGTH USING FUNGTION RV 40 $P L=R V(1)$

C----ADD NEW PELLET LENGTH TO CURRENT STACK LENGTH $\mathbf{S L}=\mathbf{S L}+\mathbf{P L}$

C---- CHECK CURRENT STACK LENGTH AGAINST LOWER LIMIT.

C IF SHORT, GET ANOTHER PELLET

IF (SL.LT.SLMIN) GO TO 40

C----CHECK CURRENT STACK LENGTH AGAINST UPPER LIMIT

C IF WITHIN TOLERANCE, STACK IS COIPLETED IF (SL.LE.SLMAX) GO TO 60

C-----STACK IS TOO LONG

C DETERMINE THE NUMBER OF TRIES AT COPILETING THE STACK NTRY $=$ NTRY +1

IF (NTRY. GT. NTRIES) GO TO 50

C-----SUBTRACT LAST PELLET AND GET A NEW ONE $\mathbf{S L}=\mathbf{S L}-\mathrm{PL}$ GO TO 40

C-----EXCEEDED THE MAXIMUM NUMBER OF ATTEMPTS

C INCREMENT THE FAILURE COUNTER BY ONE AND START A NEW STACK 50 NFAIL=NFAIL+1 GO TO 30

C-- - SAVE THE RESULTING STACK LENGTH IN THE A ARRAY AT INDEX I 1 $60 \mathrm{~A}(\mathrm{I} 1)=\mathrm{SL}$

C----PRINT THE NUMBER OF FAILUPES WRITE $($ IT, 70) NFAIL

70 FORPAT $/, 1 X, 110,31 \mathrm{H}$ STACKS FAILED TO BE COHPLETED.)

C-- - - END OF PASS 2

$$
\text { RETURN }
$$

= METER SIMULATION FOR STACKING. PELLETS

$100,0,1,1,1000,2$

$1,84,0,0.368,5$

101 , NORMAL, $0.615,0.002,0.615,0.020 \%$ PELLET LENGTH DISTRIBUTION

201 , ( STACK LENGTH DISTRIBUTION)

30 ! : ( STALK LENGTH)

$\therefore 1$, ( INCHES)

$501,0.05$ 
LISTING OF INPUT DATA FOR CASE 1

\section{= METER SIMULATION FOR STMCKING PELLETS}

$100,0,1,1,1000,2$

0001

0002

0003

0004

0004

0006

0007

$1,84.0,0.368,5$

101 , NORMAL, $0.615,0.002,0.615,0.020$ PELLIT LENGTH DISTRIBUTION 201, ( STACK LENGTH DISTRIBUTION)

301 , ( STACK LENGTH

401 , ( I NCHES)

$501,0.05$

KEY VARIABLES

$\begin{array}{rrr}\text { SIGMA }= & 4.0000 \\ \text { ICARRY }= & 0 \\ \text { IN IT }= & 0 \\ \text { INRAN }= & \mathbf{1 5 2 5 8 7 8 9 0 6 2 5 0} \\ \text { ICASE }= & -1 \\ \text { IDDATA }= & 35 \\ \text { CLASES }= & 101 \\ \text { IND1 }= & 36 \\ \text { IND2 }= & 37 \\ \text { IND3 }= & 38 \\ \text { IND4 }= & 50 \\ \text { IND5 }= & 51 \\ \text { IND6 }= & 1651 \\ \text { IAVAIL }= & 1355 \\ \text { AMOUNT }= & 31841 \\ \text { IT }= & \text { OUTPUT } \\ \text { NER }= & 0 \\ \text { DISPAR }= & 12 \\ \text { NIN }= & 1 \\ \text { NOUT }= & 1 \\ \text { NREPS }= & 1000 \\ \text { NOP }= & 2\end{array}$

- STACKS FaILED TO BE COMPLETED.

I SAMPLE PROBLEM 3)

METER 
METER SIMULATION FOR STACKING PELLETS

(SAMPLE PROBLEM 3)

CASE 1

STACK LENGTH DISTRIBUTION

CLASS
1
2
3
4
5
6
7
8
9
16
11
12
13
14

\begin{tabular}{|c|c|}
\hline $\begin{array}{c}\text { ( UNITS ARE } \\
\text { HINIMUM } \\
83600 \theta+02 \\
836590+02 \\
83700 \theta+02 \\
83750 \theta+02 \\
83800 \theta+02 \\
83859 \theta+02 \\
83909 \theta+02 \\
83959 \theta+02 \\
84609 \theta+02 \\
84059 \theta+02 \\
84109 \theta+02 \\
84150 \theta+02 \\
84200 \theta+02 \\
84250 \theta+02\end{array}$ & $\begin{array}{r}\text { I NCHES } \\
\text { MAXI MUM } \\
836500+02 \\
837000+02 \\
837500+02 \\
838000+02 \\
838500+02 \\
839000+02 \\
839500+02 \\
849000+02 \\
840500+02 \\
841000+02 \\
841500+02 \\
842000+02 \\
842500+02 \\
843000+02\end{array}$ \\
\hline
\end{tabular}

$\begin{array}{rc}\text { COUNTS } & P(X) \\ 298 & 298000+00 \\ 344 & 344000+00 \\ 7 & 700000-02 \\ 0 & 000000+00 \\ 0 & 000000+00 \\ 0 & 000000+00 \\ 0 & 000000+00 \\ 0 & 000000+00 \\ 9 & 000000+00 \\ 0 & 000000+00 \\ 0 & 000000+00 \\ 9 & 900000-02 \\ 340 & 340000+00 \\ 2 & 200000-02\end{array}$

$N=1000$

MINIYUM $=836320+02$

MAXIYUM $=842506+02$

MEAN $=838573+02$

VARI AKCE $=761311-01$

STANDARD DEVIATION $=275919+00$

COEFF ICIENT OF SKEWNESS $=620096+00$

$* * * * * * * * * *$ NOMINAL VALUE $=840000+02$

INRAN $=7802381922$ 


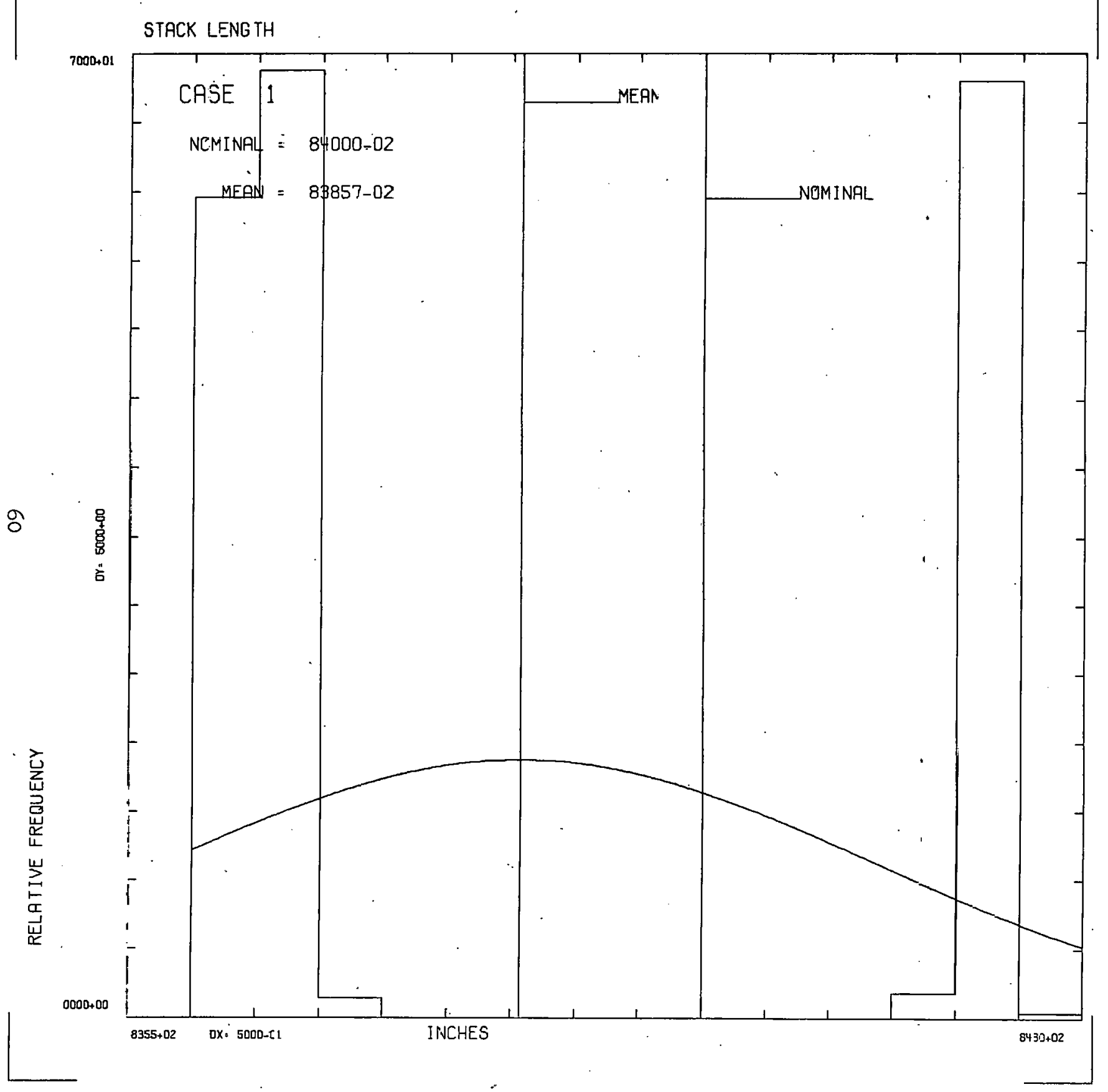




\section{D. Suggestions for Verifying Simulations}

Since the use of METER requires some new and untested programing for each application, the user must be aware of his obligation to verify his coding through sufficient testing before munning actual problems. This is a serious and often difficult task, for how does one procede to verify a computer program which by its very design is intended to produce random results? The subroutines and functions which form the MEIER package have been extensively tested and are judged to operate as intended. The user's programming can be verified in two ways.

Test the programming outside of METER if possible. By writing a small driver program to supply input to the SIM subroutine and print values of variables computed by SIM, results can be compared to hand calculations. Occasionally, a verified computer program might be used as the SIM subroutine and the verification of that program would be valid.

To test the SIM subroutine within METER, make use of the point distribution; that is, specify on the distribution input cards. (1ss) that all input distributions consist of constant values. Run a few histories and verify that METER always produces identical and correct answers at each history. This will ensure that the user's coding has not interferred with the coding in the METER package. By all means be thorough in the verification process. Once METER is used to generate supposedly meaningful results, it may be impossible to distinguish coding errors from the variability of the results.

\section{SOME METER APFLICATIONS REIATED TO LWBR}

The METER program was developed for the LWBR program when assessments of design and manufacturing uncertainties vere needed. Several of 
the Monte Carlo simulations programmed for LWBR were intended to test the feasibility of proposed fuel rod assembly procedures. Some of the METER applications related to LWBR will be sumarized to provide a feel for the types of problems which can be dealt with using Monte Carlo techniques.

III. A. Fuel Rod Binary $\left(\mathrm{UO}_{2}-\mathrm{ThO}_{2}\right)$ Stack Lengths

The first use of METER in the LWBR program involved the simulation of the starking of binary (uranium uxlde-thorlum oxide mLture) fucl peljots to form the binary portion of a fuel rod. Sample problem 3 in section III.C. 4 demonstrates the pellet stacixine simulation. These first Monte Carlo simulations demonstrated the feasibility of meeting the binary stack length tolerances given expected pellet length distributions and provided insight into the expected binary stack length distributions. It was observed, for example, that relatively few high zone seed fuel stacks would be manufactured at the design nominal of 84 inches. Since the nominal high zune seed pellet length io $0.615+0.020$ inches, the stacks will contain elther 136 or 137 pellets and the stack length distribution should peak near 83.640 inches and 84.255 inches with a valley near 84 inches. The METER sirnulation of sample problem 3 showed peals at histogram class $2(83.65-83.70)$ and at class $13(84.20-84.25)$.

\section{B. Fuei Rod Fissile Content}

The Inftial binary stack simulation was soon expanded to permit the independent random variables to include all of the pellet parameters (length, diameter, end dishes, tapers, chips, density, and uranium isotupic content) so that the resulting distributions of rod fissile content and fissile linear 
density (fissile grams per unit length) could be estimated. At the time that this simulation was programmed, the fissile content of the LVBR core was controlled by specifications on the fissile content (grams) in each of the various fuel rod types in the core. Because of thermal and hydraulic considerations it was decided that a control on fissile content per unit length would be more appropriate. METER was used to examine the fissile grams per unit length distributions as functions of the distributions of the pellet properties. It was demonstrated that the tolerances in relative percent which had been specified on rod fissile content could be applied to fissile content per unit length with a high degree of assurance that the new requirements could be satisfied at a high yield rate (few rejects).

\section{C. Thoria Shim Pellets}

A simulation was programmed to examine the procedure for assembling fuel rods. In this case the independent random variables were tubing length, bottom end cap insert length, binary pellet length, and thoria pellet lengths. Each fuel rod contains both regular thoria pellets and thoria shim pellets to precisely position the binary stack within a fuel rod and to control the size of the plenum region. This simuation was used to test the concept of shim pellets and to provide some insight into categories of shim pellet lengths needed to successfully load fuel rods.

\section{D. Flow Area Between Fuel Rods}

MEIER was used to study the allowances on LWBR thermal capability for blanket fuel rod ovality, shrinkage (collapse of the cladding against the fuel pellets), grooving (additional collapse of the cladding at pellet interfaces because of pellets with tapered ends) and wear (at points where the rods 
contact the grids influencing the rod locations). The functional relationship between the subchannel flow area and the ovality, shrinkage, grooving, and wear of fuel rods for constant rod outer diameter and rod-to-rod spacing was programmed into a subroutine and used in METER. METER generated a distribution, a mean, and a standard deviation for the flow area based on input distributions for the independent variables. The minimum flow area was then computed to be the mean minus two standard deviations (95\% confidence interval). This use of METER produced a more realistic estimate of the eff'ect on thermal pert'ormance of ovality, shrinkage, groovinğ, and wear thă would have been obtained from worst case values of the independent variables.

IV. SUMMARY

METER is a system of computer subroutines and functions which by itself is an incomplete computer program. The MELER user must describe the functional relationship between one or more independent random variables and one or more dependent random variables in a subroutine (SIM) which when appended to the METER package produces a working program. METER will process input, generate random numbers, calculate random results, and display the results.

This Monte Carlo tool has found mimerous applications related to LWBR at the Bettis Atomic Power Laboratory. It has the potential for dealing with statistical and probablistic problems which are difficult to solve through analytical methods. METER can be used to compute the frequency distribution of a simple algebraic expression or it can be used to simulate a complex mamufacturing process. As an excercise, it can be used to demonstrate and test theories of statistics. In its practical use, METER can compare "worst case" evaluations to statistical evaluations with associated confidence statements. 


\section{ACKNOWLEDGEMENTS}

The author wishes to thank $\mathrm{Mr}$. David $\mathrm{H}$. Jones for his insight in identifying the need for and the usefulness of a Monte Carlo program for simulating LWBR manufacturing processes and Mr. R. K. Kunita and Mr. K. D. McWilliams for their examples of the use of METER for LWBR in sections III.B and III.D.

REFERENCE

W. R. Cadwell, "Reference Manual - Bettis Programming Environment," WAPD-TM-1181, September 1974. 
i. Program Name (and Title): MPTER, A Monte Carlo Simulation System

2. Computer and Language (s): CDC-6600, FORTRAN

3. Problem Solved: METER is a Monte Carlo computer program which can be used to simulate the interaction between independent random variables and their effects on dependent random variables. The program generates random values f'or independent variables according to input instructions, computes the dependent variable values given the users subroutine (SIM) for computing the dependent variables, and displays results.

4. Restrictions on Complexity of Problem: Depends on size of SIM subroutine, number of variables, and number of histories.

5. Related añ Auxiliary Programs: ITone

6. Typical Running Time: Variable - a few seconds for simple simulations with 1,000 - 10,000 histories.

7. References:

(a) W. R. Cadwe].J, "Reference Manual - Bettis Prograrming Environment," WIAPD-TM-118., September 197/.

(b) B. R. Beaudoin, "Monte-Carlo Simlation Using the METER System - With Apolications Related to LVBR," WAPD-TM-1288, October 1976.

3. Unusual Features of the Program: User programs the computation of dependent variables given independent variables into his own subroutine, appends the subroutine to MLTER, then executes METER.

9. Status: Production

10. Machine Requirements: CDC-6600 or equivalent.

11. Operating System or Monitor: SCOPE

12. Other Programming, Restrictions, or Operating Intormation: Usès the Bett1s Input Package described in Reference (a).

13. Name and Estabilishment of Author:

B. R. Beatudoin

Westinghouse Electric Corporation

Bettis Atomic Power Laboratory

West Mifflin, Pennsylvania 15122 